\title{
A connectionist modeling study of the neural mechanisms underlying pain's ability to reorient attention
}

\author{
Robert Dowman ${ }^{1}$ - Benjamin Ritz $^{2}$ - Kathleen Fowler ${ }^{2}$
}

Published online: 25 April 2016

(C) Psychonomic Society, Inc. 2016

\begin{abstract}
Connectionist modeling was used to investigate the brain mechanisms responsible for pain's ability to shift attention away from another stimulus modality and toward itself. Different connectionist model architectures were used to simulate the different possible brain mechanisms underlying this attentional bias, where nodes in the model simulated the brain areas thought to mediate the attentional bias, and the connections between the nodes simulated the interactions between the brain areas. Mathematical optimization techniques were used to find the model parameters, such as connection strengths, that produced the best quantitative fits of reaction time and event-related potential data obtained in our previous work. Of the several architectures tested, two produced excellent quantitative fits of the experimental data. One involved an unexpected pain stimulus activating somatic threat detectors in the dorsal posterior insula. This threat detector activity was monitored by the medial prefrontal cortex, which in turn evoked a phasic response in the locus coeruleus. The locus coeruleus phasic response resulted in a facilitation of the cortical areas involved in decision and response processes timelocked to the painful stimulus. The second architecture involved the presence of pain causing an increase in general arousal. The increase in arousal was mediated by locus coeruleus tonic activity, which facilitated responses in the cortical areas mediating the sensory, decision, and response processes involved in the task. These two neural network
\end{abstract}

Robert Dowman

rdowman@clarkson.edu

1 Department of Psychology, Clarkson University, 8 Clarkson Ave, Potsdam, NY 13699-5825, USA

2 Department of Mathematics, Clarkson University, Potsdam, NY, USA architectures generated competing predictions that can be tested in future studies.

Keywords Involuntary attention $\cdot$ Pain $\cdot$ Connectionist modeling $\cdot$ Locus coeruleus $\cdot$ Event-related potentials (ERPs) . Norepinephrine

The ability to detect a biologically significant stimulus that occurs outside the focus of attention and redirect attention toward it is critical for survival (Bishop, 2008; Corbetta, Patel, \& Shulman, 2008; Corbetta \& Shulman, 2002; Norman \& Shallice, 1986). Pain is an excellent example of such a stimulus, given that it signals the presence of a threat to the body - that is, something that can or has the potential to cause damage (International Association for the Study of Pain Task Force on Taxonomy, 1994). Indeed, pain or the threat of pain plays an important role in escaping from and/or avoiding noxious stimuli and facilitating recuperation (Basbaum \& Jessel, 2000; Goldberg et al., 2012). Evidence supporting the notion that pain facilitates the reorienting of attention has been reported in studies investigating the ability of a task-irrelevant painful stimulus to interfere with a primary task, as shown by an increase in primary-task errors and/or reaction times. Whether or not pain interferes with the primary task depends on the characteristics of the painful distractor. Pain that is strong or novel, has an abrupt onset, is perceived as threatening, and/or is unexpected is most effective at interfering with a primary task (Crombez, Eccleston, Baeyens, \& Eelen, 1998; Eccleston, 1994; Eccleston \& Crombez, 1999; Vancleef \& Peters, 2006; Van Damme, Crombez, Van Nieuwenborgh-De Wever, \& Goubert, 2008).

The psychophysical evidence described above demonstrates that under the appropriate conditions, pain can shift attention away from some other stimulus or task and toward 
itself. However, the neural mechanism underlying this process is not clear (Legrain, Iannetti, Plaghki, \& Mouraux, 2011; Van Damme, Legrain, Vogt, \& Crombez, 2010). We have explored this mechanism using a cross-modal endogenous cuing paradigm (Dowman, 2007a, b, 2014; Dowman \& ben-Avraham, 2008). In these studies, participants performed cued visual color discrimination and somatosensory intensity discrimination tasks. The target stimuli in the somatosensory discrimination task involved either nonpainful (Dowman, 2007b, 2014) or painful (Dowman, 2007a, 2014) electrical stimulation of the sural nerve. The cue signaled the participant to voluntarily direct their attention to either the visual or the somatosensory target modality. The target sensory modality was correctly cued on most ( $75 \%$; validly cued) but not all ( $25 \%$; invalidly cued) trials. The behavioral reaction time data obtained in those studies suggest that the painful sural-nerve electrical target is able to shift attention away from the invalidly cued visual target modality and toward itself more quickly than is a nonpainful sural-nerve electrical target (Dowman, 2014; Dowman \& ben-Avraham, 2008). Furthermore, eventrelated potentials (ERPs) recorded during those studies suggest a possible neural mechanism: An unattended somatic threat is detected by somatic threat detectors located in the dorsal posterior insula. The somatic threat detector activity is monitored by the medial prefrontal cortex, which in turn signals the lateral prefrontal cortex to redirect attention toward the potential threat (Dowman, 2014; Dowman \& benAvraham, 2008).

We have explored this threat-detection-and-reorienting hypothesis using connectionist modeling (Dowman \& ben-Avraham, 2008). Connectionist models involve the accumulation of information through a network of nodes, where the nodes represent the brain areas involved in the cognitive process, and the connections between the nodes represent the interactions between the brain areas (Bogacz \& Cohen, 2004; Cohen, Dunbar, \& McClelland, 1990). Different model architectures are constructed to test different hypotheses concerning the brain areas responsible for the cognitive process. Connectionist modeling has several advantages. First, it requires a rigorous, explicit, and quantitative formulation of the cognitive process that can be directly translated to brain mechanisms (Bogacz \& Cohen, 2004). Second, it permits the comparison of alternative hypotheses to determine which provides the best fit of the experimental data, and by implication which is more likely to be correct (Bogacz \& Cohen, 2004; Dowman \& ben-Avraham, 2008). Note, however, showing that an architecture does fit the data does not prove that it is correct. Third, connectionist modeling provides the opportunity to run virtual experiments and/or measurements that have not yet been done experimentally. These in turn can lead to unanticipated, novel predictions that can be tested in the laboratory (e.g., Deco \& Rolls,
2005; Yeung et al., 2004). Experiments that verify the predictions provide further evidence for the feasibility of the architecture.

Our previous connectionist modeling study (Dowman \& ben-Avraham, 2008) demonstrated the feasibility of the somatic threat-detection-and-reorienting hypothesis suggested by our experimental work. The modeling study also suggested that the reorienting of attention is directed mainly toward decision and response processes, and not sensory processes. However, that study had several limitations. First, the modeling involved fitting the reaction time and ERP data elicited by the painful sural-nerve target stimuli presented in Dowman (2007a) and the nonpainful sural-nerve targets presented in Dowman (2007b). Reaction times decrease with increasing stimulus intensity (Bushnell, Duncan, Dubner, Jones, \& Maixner, 1985; Dowman, 2001, 2007a, 2014; Miron, Duncan, \& Bushnell,1989). Consequently, the different stimulus intensities used in these two studies introduced a stimulus intensity confound. Furthermore, the tasks involving the somatosensory stimuli in the two studies were different: The painful sural-nerve stimuli were targets in a pain-rating task (Dowman, 2007a), whereas the nonpain stimuli were used in an intensity discrimination task (Dowman, 2007b). These confounds prevented a direct comparison between the experimental and simulated reaction times. Rather, the invalidly cued-validly cued reaction time difference (validity effect) was used instead.

Second, the model parameters were adjusted manually to provide a good fit for one of the model architectures, and were held constant for the other architectures. It is possible that one architecture could have provided the best fit of the experimental data with one set of parameters, whereas another architecture might have provided an even better fit had another parameter set been used. Furthermore, manual adjustment of the model parameters only permitted qualitative fits of the model and experimental data - that is, demonstrating that the simulated and experimental reaction time and ERP data went in the same direction - and did not provide quantitative fits. Third, the modeling only compared architectures that were essentially variations of the somatic threat-detection-and-reorienting hypothesis, and did not include architectures that involved other hypotheses.

The goal of the present study was to replicate and extend the Dowman and ben-Avraham (2008) connectionist modeling study by addressing the limitations noted above. First, we used recently obtained experimental data reported in Dowman (2014) that allowed a direct comparison between the experimental and simulated reaction times. These new data suggest that a nonpainful sural-nerve target activates somatic threat detectors when it is presented in a pain context (i.e., is paired with a painful sural-nerve target; Dowman, 2014), but not when it is presented in a pain-absent context (i.e., is paired with another, nonpainful sural-nerve target: Dowman, 2007b; 
see Dowman, 2014). This allowed us to compare potentially threatening (i.e., a nonpainful sural-nerve target presented in a pain context) and nonthreatening (i.e., a nonpainful suralnerve target presented in a pain-absent context) sural-nerve target stimuli whose stimulus intensities were comparable. Likewise, the same intensity discrimination task was used in the pain and pain-absent contexts. Hence, comparing the reaction time and ERP data obtained from a nonpainful suralnerve target presented in pain and pain-absent contexts significantly reduced or eliminated the stimulus intensity and task difference confounds present in the Dowman and benAvraham modeling study. These new results also allowed us to determine whether the modeling results reported by Dowman and ben-Avraham would generalize to a different data set.

Second, we used mathematical optimization techniques to search for the model parameters that provided the best quantitative fit of the experimental reaction time and ERP data. This permitted a fairer comparison between the different model architectures. Third, we included two model architectures that are qualitatively different from the somatic threatdetection-and-reorienting hypothesis. The model architectures examined in Dowman and ben-Avraham (2008) were based on the hypothesis that the somatic threat signal generated by the medial prefrontal cortex is sent to the lateral prefrontal cortex via direct anatomical connections. These connections have been demonstrated in a number of studies and are a basic component of attentional control implemented in connectionist models of response conflict (see Carter \& van Veen, 2007, for a review). An alternative might involve the somatic threat signal from the medial prefrontal cortex eliciting a phasic response in the locus coeruleus. Salient, biologically significant, and/or unexpected stimuli have been shown to elicit a phasic response in the locus coeruleus, which results in a phasic increase in the release of norepinephrine throughout the cerebral cortex (Aston-Jones \& Cohen, 2005; Berridge \& Waterhouse, 2003; Sara \& Bouret, 2012). The end result is a phasic increase in the gain of the cortical neurons that facilitates cortical responses that are time-locked to the evoking stimulus (Aston-Jones \& Cohen, 2005; Nieuwenhuis, AstonJones, \& Cohen, 2005). The timing of the gain increase will largely affect decision (e.g., stimulus-response mapping) and response processes (Aston-Jones \& Cohen, 2005), which in theory would reduce the reaction time to an invalidly cued (i.e., unexpected) potentially threatening somatic target.

We also examined an architecture simulating an increase in tonic arousal. It is reasonable to assume that tonic arousal levels will be higher in a pain context than in a pain-absent context. An increase in arousal is mediated by tonic activity in the locus coeruleus (Aston-Jones \& Cohen, 2005; Berridge \& Waterhouse, 2003; Sara, 2009), which in turn results in a tonic increase in the gain of the cortical neurons involved in the task, including those involved in sensory, decision, and response selection processes. Here we investigated whether an increase in tonic arousal can explain the faster reaction times for an invalidly cued nonpainful sural-nerve target stimulus that was presented in a pain context versus a pain-absent context.

\section{Method}

\section{Experimental data}

Task The experimental data used here were taken from the cross-modal endogenous-cuing studies reported by Dowman (2007b, 2014). In each study, the participants performed two tasks presented in random order with equal probabilities: a visual color discrimination task and a somatic intensity discrimination task. For the color discrimination task, participants determined whether a red or a yellow LED located next to their right ankle was lit. The somatic intensity discrimination task involved determining whether a low- or a highintensity electrical stimulus was delivered to the right sural nerve at the ankle. In Dowman (2007b), both of the suralnerve targets were nonpainful (pain-absent context), and in Dowman (2014), one was nonpainful and the other produced a moderately strong pain sensation (pain context). A symbolic cue given at the beginning of each trial signaled participants to direct their attention toward one of the target stimulus modalities. The interval between the cue offset and target onset was $1,500 \mathrm{~ms}$, which allowed ample time for any involuntary attention effects elicited by the cue to dissipate and for the participants to voluntarily direct their attention toward the target stimulus modality (Müller \& Rabbit, 1989). The target sensory modality (visual vs. somatosensory) was correctly cued on a randomly determined $75 \%$ of the trials (validly cued condition) and was incorrectly cued on the remaining $25 \%$ of the trials (invalidly cued condition). In the invalidity cued suralnerve condition, the participants had to shift their attention away from the cued visual target modality and toward the sural-nerve target, and vice versa for the invalidly cued color target. The participants were given a total of 640 trials, with 120 validly cued and 40 invalidly cued trials for each target type. The data modeled here were the grand averages for the different cue validity and pain context conditions. That is, the data were averaged across all trials in each condition and then across all participants.

As we noted above, evidence reported by Dowman (2014) suggests that a nonpainful sural-nerve target stimulus will elicit behavioral reaction time and ERP evidence for activation of somatic threat detectors when it is presented in a pain context (paired with a painful sural-nerve target; Dowman, 2014), but not when it is presented in a pain-absent context (paired with another nonpainful sural-nerve target; Dowman, 2007b). Hence, here we used the reaction time and ERP data elicited by the strongest nonpainful sural-nerve target 
presented in the pain-absent context with the nonpainful suralnerve target presented in the pain context. The stimulus levels for these two nonpainful sural-nerve stimuli were comparable, and the same intensity discrimination task was used in the pain and pain-absent contexts. Hence, using these data allowed us to avoid the stimulus intensity and task confounds present in the painful versus nonpainful sural-nerve target levels modeled in Dowman and ben-Avraham (2008).

Reaction time data The grand average reaction times for the nonpainful sural-nerve target presented in the pain and painabsent conditions are shown in Table 1. Reaction times were faster in the pain than in the pain-absent context [pain context main effect: $F(1,40)=21.33, p<.0001]$, and this effect was largest in the invalidly cued condition [Pain Context $\times$ Cue Validity interaction: $F(1,40)=25.06, p<.0001$; see Dowman, 2014]. These data are consistent with the idea that participants are better able to detect and reorient attention toward an unattended nonpainful sural-nerve target stimulus when it is presented in the pain context rather than the pain-absent context.

ERP data Our previous work has reported three components of the ERP evoked by painful sural-nerve target stimuli whose amplitudes were larger in the invalidly cued than in the validly cued condition of the cross-modal cuing paradigm (Dowman, 2007a, 2014; Dowman \& ben-Avraham, 2008). The earliest of these components is a bilateral negative potential recorded from the fronto-temporal scalp at about $150 \mathrm{~ms}$ poststimulus, where the negativity is larger on the side contralateral to the evoking stimulus (the contralateral temporal negativity; CTN). The second component is a negative potential located at the fronto-central scalp (FCN) that overlaps temporally with the $\mathrm{CTN}$, and the third is the P3a component. We have shown that pain ratings are lower in the invalidly cued than in the validly cued condition (Dowman, 2001, 2007a), which makes it very unlikely, therefore, that the increases in the CTN, FCN, and $\mathrm{P} 3 \mathrm{a}$ amplitudes in the invalidly cued condition are related to sensory processes. Rather, the increase may reflect their roles in detecting and reorienting attention toward a painful sural-nerve target.
The response properties of their neural generators are consistent with this hypothesis. Our dipole source localization and intracranial recording studies suggest that the CTN is generated by temporally overlapping activity in the second somatosensory cortex and the adjacent dorsal posterior insula (Dowman \& Darcey, 1994; Dowman, Darcey, Barkan, Thadani, \& Roberts, 2007), both of which are located in the superior bank of the Sylvian fissure. The dorsal posterior insula is activated by stimuli that can be considered threats to the body, such as noxious stimuli, air hunger, and deviations of body temperature away from homeostasis (Craig, 2002, 2003). These response properties are consistent with the idea that the dorsal posterior insula is involved at least in part in a somatic threat detection process. Likewise, the response properties of the CTN suggest that its validity effect is specifically related to somatic threats: Its amplitude is larger in the invalidly than in the validly cued condition for painful targets and for nonpainful targets presented in a pain context (Dowman, 2007a, 2014), but not for nonpainful targets presented in a pain-absent context (Dowman, 2007b). The cue validity effect for the CTN evoked by nonpainful targets presented in a pain context presumably involves sensitization of the somatic threat detectors to the nonpainful stimuli (Dowman, 2014).

The cue validity effects for the FCN and P3a, on the other hand, were not specific to pain, but also occurred for nonpainful targets presented in a pain-absent context (Dowman, 2007b). This result suggests that the FCN and P3a play a more general role in attentional control. This observation is consistent with the response properties of their neural generators. A large body of evidence has shown that the P3a recorded from the anterior scalp indexes prefrontal cortex activity related to the orienting response elicited by any unexpected, infrequent, and/or novel stimulus (see Friedman et al., 2001; Knight, 1996; Polich, 2003, for reviews). Our dipole source localization and intracranial recording studies suggest that the FCN is generated in part by the medial prefrontal cortex, specifically the dorsal anterior cingulate cortex and the overlying presupplementary motor area (Dowman, 2001, 2004; Dowman \& Darcey, 1994; Dowman et al., 2007; Dowman, Glebus, \& Shinners, 2006).

Table 1 Experimental reaction time and sural-nerve event-related potential component peak amplitudes

\begin{tabular}{|c|c|c|c|c|c|c|c|c|}
\hline & \multicolumn{2}{|c|}{ Reaction Time (ms) } & \multicolumn{2}{|c|}{ CTN $(\boldsymbol{\mu} \mathrm{V})$} & \multicolumn{2}{|l|}{$\mathrm{FCN}(\mu \mathrm{V})$} & \multicolumn{2}{|l|}{$\mathrm{P} 3 \mathrm{a}(\mu \mathrm{V})$} \\
\hline & Pain Abs & Pain & Pain Abs & Pain & Pain Abs & Pain & Pain Abs & Pain \\
\hline $\mathrm{VC}$ & 684.5 & 566.4 & -5.7 & -4.9 & -0.7 & -2.5 & 5.9 & 5.8 \\
\hline IC & 809.5 & 616.2 & -5.9 & -5.6 & -1.9 & -3.1 & 6.6 & 6.0 \\
\hline$\% \uparrow I C$ experimental & - & - & 3.5 & 14.4 & 171.4 & 26.4 & 11.9 & 3.4 \\
\hline$\% \uparrow I C$ modeled & - & - & 0.0 & 14.4 & - & - & - & - \\
\hline
\end{tabular}

Abbreviations: Pain Abs = pain-absent context; Pain = pain context; VC = validly cued condition; $\mathrm{IC}=$ invalidly cued condition; $\% \uparrow \mathrm{IC}=$ percent increase in the invalidly cued condition; $-=$ data were not modeled. Percent increases in the invalidly cued condition that were statistically significant $(p<.05)$ are given in bold 
One of the roles of the medial prefrontal cortex is monitoring for situations that require a change in attentional control and signaling the lateral prefrontal cortex to execute the change (see Botvinick, Braver, Barch, Carter, \& Cohen, 2001; Carter \& van Veen, 2007; and Yeung, Botvinick, \& Cohen, 2004, for reviews). Clearly, an unattended somatic threat qualifies as a situation that requires a change in attentional control (Bishop, 2008; Eccleston \& Crombez, 1999; Van Damme et al., 2010).

Together, these electrophysiological data present a possible mechanism explaining how a potential somatic threat is detected and reorients attention: An unattended somatic threat is detected by somatic threat detectors located in the dorsal posterior insula. The somatic threat detector activity is monitored by the medial prefrontal cortex, which in turn signals the lateral prefrontal cortex to redirect attention toward the potential threat.

The grand average ERP waveforms and peak amplitudes for the CTN, FCN, and P3a elicited by the nonpainful suralnerve target presented in the pain context (Dowman, 2014) and by the highest-level nonpainful sural-nerve target presented in the pain-absent context (Dowman, 2007b) are shown in Fig. 1 and Table 1. Although the FCN and P3a amplitudes were larger in the invalidly cued than in the validly cued condition, the increase in the invalidly cued condition for these components is clearly not consistent with the greater increase in the pain context predicted by the somatic threatdetection-and-reorienting hypothesis. In fact, the invalidly cued versus validly cued differences in P3a amplitudes did not reach statistical significance (Dowman, 2007b, 2014). As expected, all attempts at modeling these two components with the reaction time data failed. There are at least two potential explanations for this result. First, the FCN and/or P3a might not be involved in reorienting attention toward a potentially threatening sural-nerve target. We will present two physiologically feasible network architectures that are consistent with this alternative.

The second involves difficulties isolating the FCN and P3a generator sources that are specifically related to monitoring somatic threats and reorienting attention. Work done in our lab and elsewhere has shown that a number of different generator sources are active during the FCN and P3a component epochs (Dowman \& Darcey, 1994; Dowman et al., 2007; Friedman et al., 2001; Halgren, Marinkovic, \& Chauvel, 1998; Polich, 2007; Soltani \& Knight, 2000). The overlap in generator sources for the FCN and P3a may have prevented acceptable isolation of the attention-related FCN and P3a generator sources required for modeling. For example, the P3a amplitudes evoked by the nonpainful sural-nerve stimulus levels used here were the same in the validly and invalidly cued conditions (Table 1; Dowman, 2007b, 2014). The sural-nerve P3a did show a statistically significant increase in the invalidly cued condition for a nonpainful intensity that was lower than the one used here (Dowman, 2007b), for the painful stimulus level (Dowman, 2007a, 2014), and for the visual targets (Dowman, 2007a, b, 2014). Clearly, the P3a validity effect is not specific to painful stimuli. Inspection of the grand average sural-nerve ERP waveforms suggested that the failure to see a P3a validity effect at this stimulus level is a spurious effect related to the overlap between the preceding positive component and the P3a (see Fig. 1; Dowman, 2014). Similarly, the FCN receives extensive overlap with a positive potential whose maximum is located at the vertex and whose validity effect is the opposite of the FCN (Dowman, 2007a, b, 2014).

The percent increase in the CTN amplitude, on the other hand, is consistent with it indexing neural activity specific to potential somatic threats. Success in isolating the somatic threat activity from the CTN likely involves its second somatosensory area and dorsal posterior insula generators being less numerous and functionally complex than those for the FCN and P3a. Hence, the modeling described here restricted the ERP analysis to the CTN.

\section{Connectionist modeling}

Model architecture Different model architectures were devised to simulate different hypotheses concerning the brain areas involved in detecting and reorienting attention toward somatic threats. An example of the basic architecture is shown in Fig. 2. This architecture includes two stimulus-response pathways corresponding to the visual color discrimination and the sural-nerve intensity discrimination tasks, where each has early sensory $(\mathrm{S})$, late sensory $(\mathrm{M})$, and response $(\mathrm{R})$ nodes. The lowercase $\mathrm{v}$ and $\mathrm{s}$ denote nodes specific to the visual color discrimination and sural-nerve intensity discrimination tasks, respectively. There are feedforward excitatory connections between the early sensory and late sensory nodes, and between the late sensory and response nodes. Voluntary attentional control involves the lateral prefrontal cortex modulating the excitability of the sensory and response areas involved in the task (Cole, Yarkoni, Repovs, Anticevic, \& Braver, 2012; D’Esposito, 2007; Miller \& Cohen, 2001; Shimamura, 2000). These lateral prefrontal cortex areas are simulated by the sensory attention nodes (Av and As) and by the response attention nodes (ARv and ARs). Bidirectional excitatory connections link the attention nodes and their respective sensory and response nodes, and bidirectional inhibitory connections link the sensory attention nodes, the response attention nodes, and the response nodes.

The threat detectors for the visual and somatosensory stimuli are simulated by the Thv and Ths nodes, respectively. The sensory attention nodes have a feedforward inhibitory connection with the threat detector nodes. This feedforward inhibitory connection merely implements the larger threat detector response in the invalidly than in the validly cued condition observed in experimental work, and is not meant to represent 


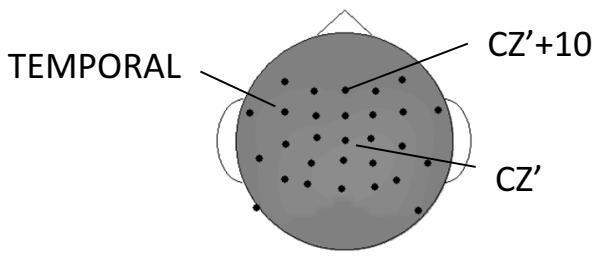

EXPERIMENTAL ERPS

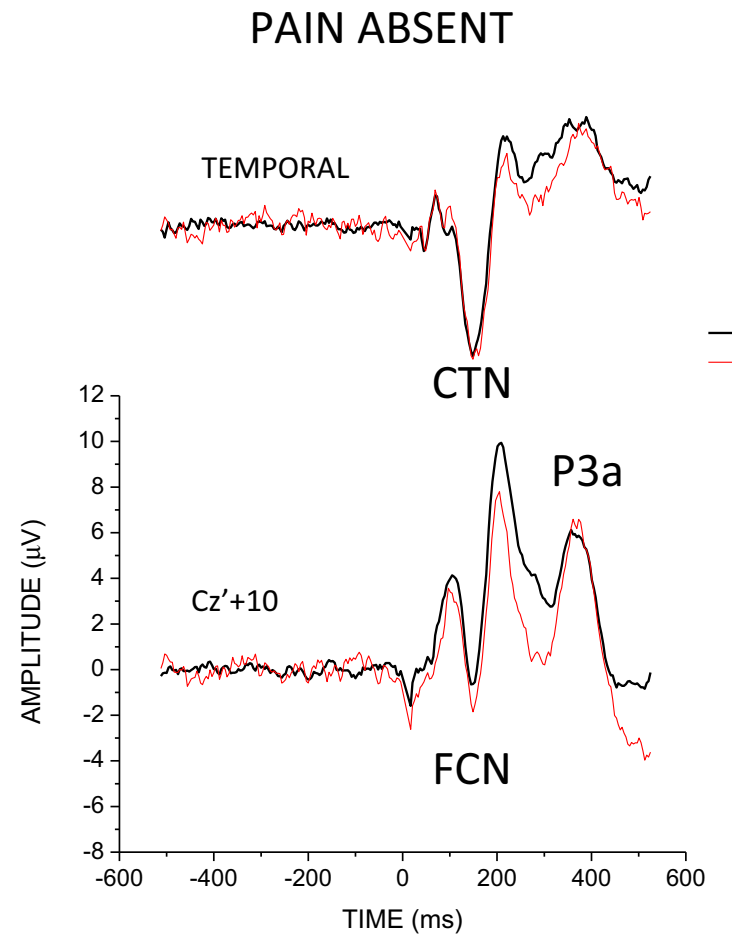

Fig. 1 Top panel: Scalp electrode locations used to record the sural-nerve event-related potential (ERP). The small solid circles identify the recording electrode locations. The interelectrode distance along both the sagittal and coronal axes was $5 \mathrm{~cm}$. CZ' $(2 \mathrm{~cm}$ posterior to the vertex position of the 10-20 electrode system) is the third electrode from the bottom along the sagittal midline. The CTN waveform was recorded from the contralateral frontotemporal location (TEMPORAL), and the FCN

the neural mechanism underlying this effect. It might, for example, involve a mismatch between an expected stimulus based on a working memory template and the stimulus input (Egner, Monti, \& Summerfield, 2010; Friston, 2005; Summerfield \& Egner, 2009; Wacongne, Changeux, \& Dehaene, 2012). The threat detectors have a feedforward excitatory connection with the node representing the medial prefrontal cortex ( $\mathrm{mPs}$ and $\mathrm{mPv}$ ), which in turn has a feedforward excitatory connection with the response attention nodes (ARv and ARs).

The symbols next to the connections (smr, smr2, asr, at, tmar, tmar2, and in) represent the connection strengths. The numerical values for these connection strengths were optimized to produce the best fit to the experimental data (see below).
PAIN

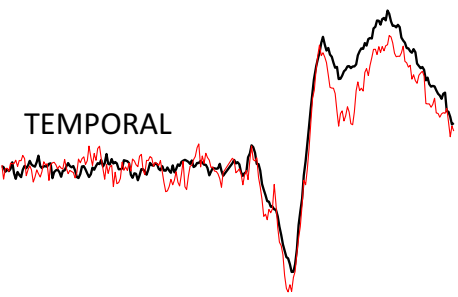

VALIDLY CUED

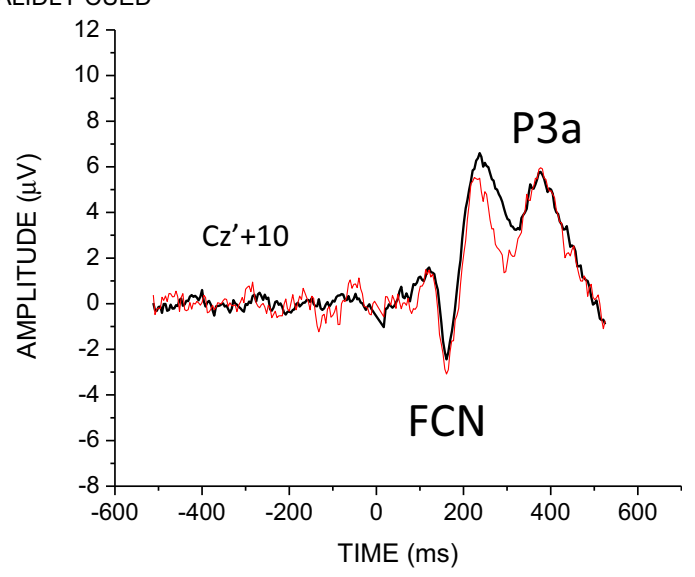

and $\mathrm{P} 3 \mathrm{a}$ were recorded from an electrode located $10 \mathrm{~cm}$ anterior to CZ' $(C Z+10)$. Bottom panels: Effects of cue validity (validly cued, invalidly cued) on the grand average sural-nerve ERP waveforms recorded during the pain-absent (bottom left panels) and pain (bottom right panels) contexts in the Dowman (2007b) and Dowman (2014) studies, respectively.

Task simulation The activation level for each node was a function of its inputs from other nodes and, when appropriate, external inputs over 60 iterations (referred to as cycles). External inputs were added to one of the sensory attention nodes on cycles $1-5$ to simulate the allocation of attention toward the target sensory modality, as directed by the cue. The validly cued sural-nerve target condition was simulated by adding an external input of 1.0 to the somatosensory attention node (As) and of 0.0 to the visual sensory attention node $(\mathrm{Av})$, and vice versa for the invalidly cued sural-nerve target condition. An external input of 1.0 was added to the somatosensory sensory node (Ss) during cycles 6-10 to simulate the presentation of the sural-nerve target stimulus. A sural-nerve stimulus that activates the somatic threat detectors (pain 
AtoM architecture

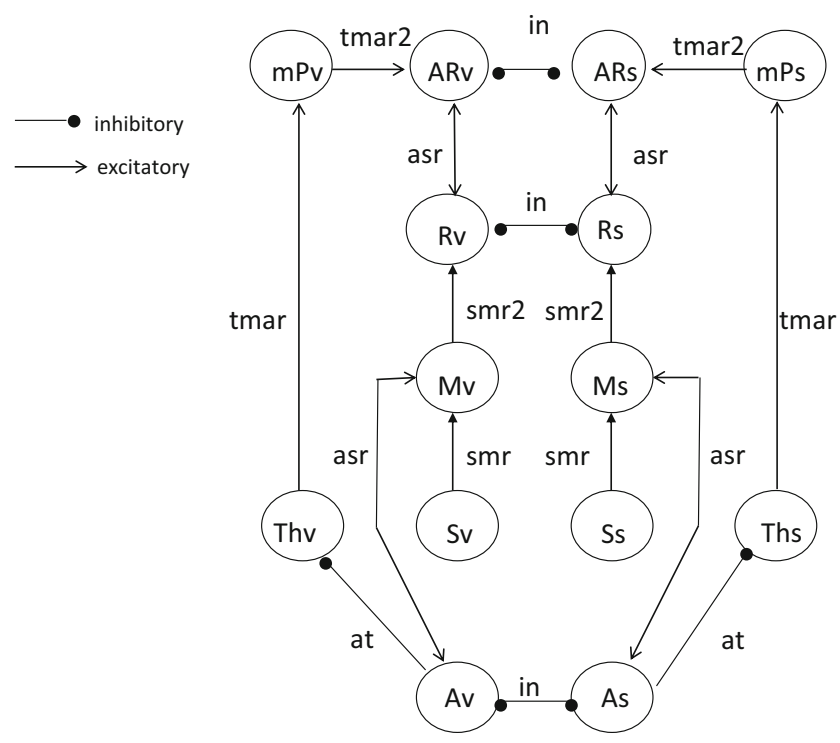

Fig. 2 AtoM architecture, simulating the somatic threat-detection-andreorienting hypothesis. The circles correspond to the nodes representing the early sensory (S), late sensory (M), and response (R) areas, the lateral prefrontal cortex areas directing attention to the sensory (A) and response (AR) areas, the threat detectors (Th), and the medial prefrontal cortex region $(\mathrm{mP})$ that monitors for the presence of threat detector activity. The lowercase $\mathrm{s}$ and $\mathrm{v}$ refer to nodes that are specific to the sural-nerve and visual tasks, respectively. The somatic threat detectors are located in the dorsal posterior insula. Feedforward excitatory connections between the nodes are represented by the single-headed arrows (arrow pointing toward the receiving node), bidirectional excitatory connections are represented by the double-headed arrows, feedforward inhibitory connections are represented by single-headed lines with closed circles (the closed circle toward the receiving node), and bidirectional inhibitory connections are represented by double-headed lines with closed circles. The symbols next to the connections (asr, smr, smr2, in, at, tmar, and tmar2) identify the connection strength parameters used in the model optimization.

context) was simulated by also adding an external input of 1.0 to the somatosensory threat detector node (Ths) during the stimulus cycles. Equal amounts of external inputs (.675) were added to both response attention nodes at each cycle to simulate the task relevance of the responses.

The experimental visual task reaction times only involved nonthreatening visual stimuli. Because the architecture is symmetrical (see Fig. 2), a nonthreatening visual stimulus will produce the same simulated reaction time as a nonthreatening somatic stimulus. Consequently, we only presented stimuli to the somatosensory side of the models. It should be noted that the experimental visual color and somatic intensity discrimination task reaction times were different (see Dowman, 2014). However, these differences can be attributed to the differences in visual and somatosensory sensory conduction times, perceptual processes, and tasks, and are not relevant to the hypotheses tested here. Furthermore, these reaction time differences can be modeled easily by using separate connection strength values for the visual and somatosensory sides of the model. However, doing so would significantly increase the computation time needed to find the optimal parameter set, with little theoretical benefit. Note that the same results were obtained when the visual threat detector (Thv) and medial prefrontal cortex $(\mathrm{mPv})$ nodes were removed from the architecture, as might be expected, given that there is little or no activation of the threat detector and medial prefrontal cortex nodes when no external inputs are added to the threat detector node (see, e.g., the mPs activation [simulating the $\mathrm{FCN}$ ] in the pain-absent context shown in Fig. 3).

The activation level for each node was computed using the following activation function:

$A_{i}=\frac{1}{1+e^{\left(C-g N_{i}\right)}}$,

where $A_{i}$ is a column vector containing the activation levels for the $K$ nodes for the $i$ th cycle; $C$ is a constant that was set to 4 ; and $g$ is the gain. $g$ was set to 1.0 for the architectures that did not involve the locus coeruleus. $N_{i}$ was defined as

$N_{i}=N_{i-1}+\left(W^{*} A_{i-1}\right)-\left(N_{i-1} * \delta\right)$,

where $N_{i}$ is a column vector containing the inputs to each of the $K$ nodes on cycle $i ; \delta$ is a scalar decay constant set to $0.1 ; W$ is a $K \times K$ matrix containing the connection strengths between the nodes; and $N_{i-1}$ and $A_{i-1}$ are the values of $N$ and $A$, respectively, during the cycle prior to cycle $i$. The initial values for $A$ and $N$ were set to zero. Since the experimental data were derived from the grand averages, a noise term was not necessary in Eq. 2.

Computing simulated reaction time and ERP values The simulated reaction time was defined as the cycle on which the response node activation equaled .2. The simulated reaction time was converted to milliseconds using the function described by Bogacz and Cohen (2004):

Reaction Time $=20 c+D$,

where $c$ is the cycle at which the activation level of the response node equals $0.2,20$ is a very rough estimate of the number of milliseconds per cycle, and $\mathrm{D}$ is a constant that accounts for the perceptual, decision, and response selection processes that are not accounted for by the model. A linear interpolation was computed when the .2 response threshold value fell between integer cycle values to obtain a more precise reaction time estimate. The simulated reaction times were directly compared to the experimental grand average reaction times when optimizing the model parameters (Table 1).

We did not compare the peak of the simulated CTN activation to the peak of the experimental grand average CTN. This decision was based on the problems associated with converting the CTN amplitude to a value that is comparable to the node activation values - namely, the magnitude of 
AtoM architecture activations

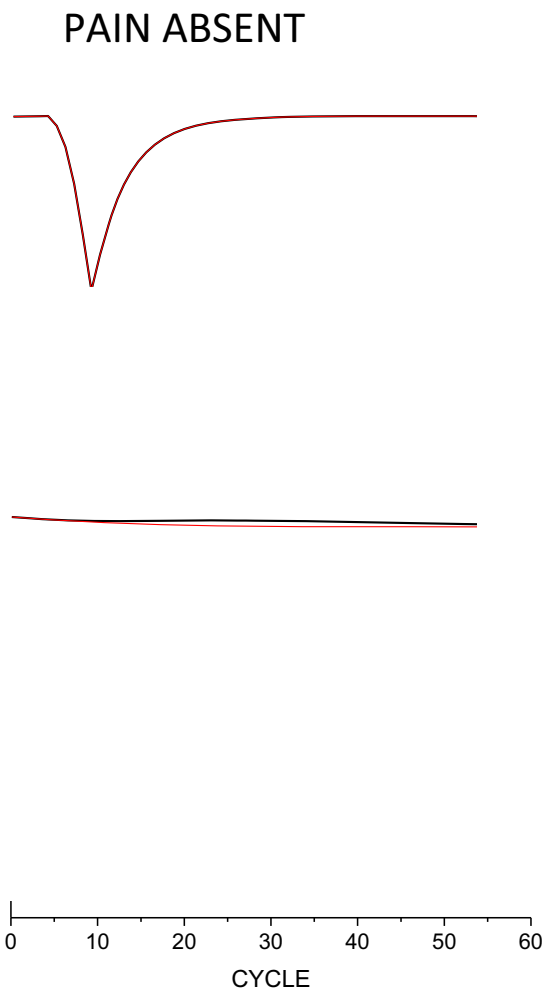

Fig. 3 Activation values for the architecture evaluating the somatic threat-detection-and-reorienting hypothesis (AtoM). Activation values are shown for nodes representing the CTN (combination of the somatosensory sensory [Ss] and threat detector [Ths] nodes; upper

activity in the neurons that generate the CTN. Ideally, one would perform a dipole source localization analysis on the ERP component and use the source magnitude as a measure of the brain activation. However, inaccuracies in the source analysis due to noise in the waveform, incorrect source starting locations, misspecification of the skull and brain dimensions, and nonfocal sources raise serious concerns about the accuracy of the computed source magnitudes (Zhang \& Jewett, 1993). Instead, we used the percent change in the grand average CTN peak amplitude in the invalidly cued relative to the validly cued condition ( $\{[$ invalidly cued - validly cued]/validly cued $\} * 100$ ), and compared it to the same statistic computed for the simulated CTN peak activation level. We validated this approach by performing a dipole sourcemodeling simulation with a radial or a tangential oriented source located $9 \mathrm{~cm}$ below the vertex, to determine how a change in source magnitude would be reflected by a change in the peak amplitude recorded from the scalp. These simulations showed that increasing the radial or tangential source magnitude $25 \%$ resulted in $25.8 \%$ and $24.6 \%$ increases in the peak amplitudes, respectively, and that a $50 \%$ increase in source magnitude resulted in $54.6 \%$ and $44.9 \%$ increases in

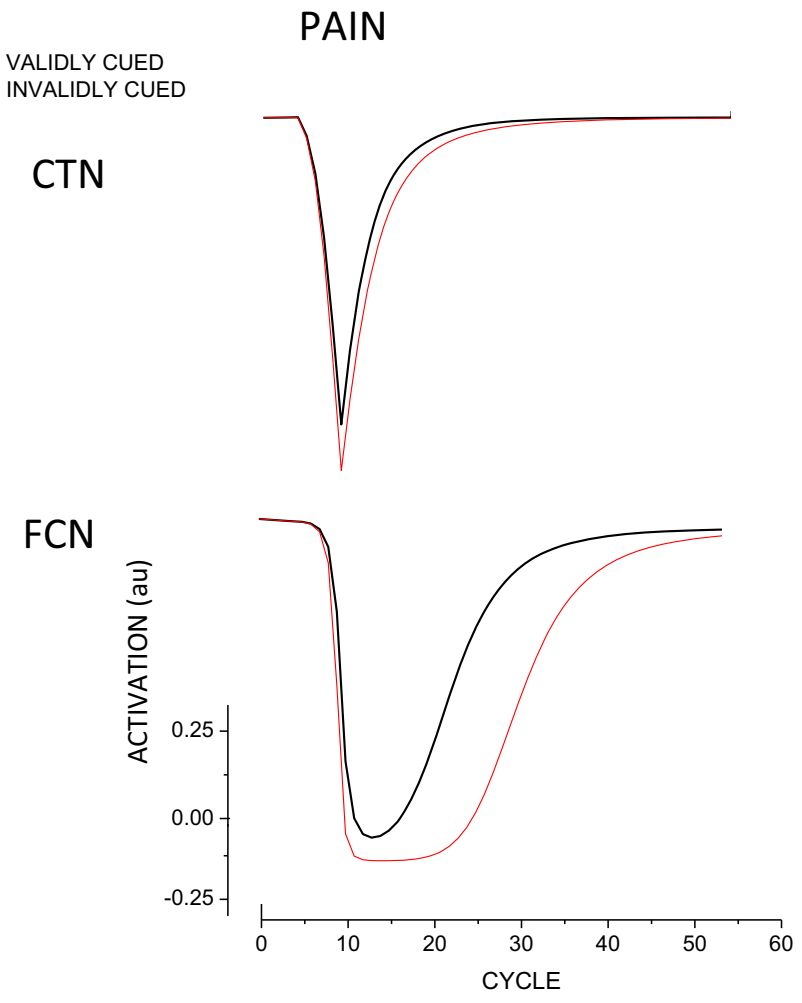

panels) and the FCN (somatosensory medial prefrontal cortex [mPs] node; lower panels) obtained in the different pain (pain-absent context, pain context) and cue validity (validly cued, invalidly cued) conditions. $\mathrm{au}=$ arbitrary units

peak amplitudes, respectively. Similar results were obtained with sources located $6 \mathrm{~cm}$ below the vertex. ${ }^{1}$ Therefore, using the percent change in the peak amplitude appears to provide an acceptable estimate of the change in the generator source activation between conditions.

As we noted above, the sural-nerve CTN appears to be largely composed of two sources: one involving innocuousrelated activity in the second somatosensory area, and painrelated (presumably including somatic threat detector) activity in the dorsal posterior insula. Hence, the CTN activation was modeled by adding the somatosensory sensory (Ss) and threat detector (Ths) node activations, as was the case in Dowman and ben-Avraham (2008). Since the difference in CTN amplitudes between the validly and invalidly cued conditions in the pain-absent condition was small and did not reach statistical significance, we used a value of $0 \%$ in the model optimization (Table 1).

\footnotetext{
${ }^{1}$ Note that the accuracy of the percent change in amplitude depends on the spatial sampling frequency of the scalp electrodes: the greater the sampling frequency, the greater the accuracy. For the values presented here, we used the scalp electrode grid used in our experimental studies (see Fig. 1; Dowman, 2007b, 2014).
} 
Model parameter optimization Optimizing the model involved searching for the parameter values that resulted in the best quantitative fit of the experimental grand average reaction time and CTN data. We used the approach recommended by Bogacz and Cohen (2004). The optimization minimized the cost function $J(V)$ :

$J(V)=\frac{1}{P} \sum_{i=1}^{P}\left(\frac{e_{i}-m_{i}}{n_{i}}\right)^{2}$,

where $V$ is a vector containing the parameters to be optimized (e.g., connection strengths); $p$ corresponds to the number of experimental measurements (statistics) that are being fit (e.g., reaction time and $\mathrm{CTN}) ; e_{i}$ is the experimental value of a given statistic; $m_{i}$ is the corresponding modeled value (which depends on $V$ ); and $n_{i}$ is the normalization factor for that statistic. The normalization factors were selected such that each statistic had about the same weight in the cost function. The value of $n_{i}$ for the reaction times equaled the average reaction time across the four pain context and cue validity conditions. Using the average value across CTN values as the normalization factor resulted in the cost function being heavily weighted toward the CTN. We found that setting $n_{i}$ to 1,000 for the CTN data resulted in the reaction time and CTN values having roughly equal weights in the cost function.

The connection strengths (e.g., smr, smr2, asr, at, tmar, tmar2, and in for the architecture shown in Fig. 2) and the constant used to correct the simulated reaction times for perceptual, decision, and response processes not accounted for by the model (D in Eq. 3) were optimized for each architecture. Including $\mathrm{D}$ in the optimization avoided the tedious and timeconsuming task of manually searching for a value that would work for a given set of reaction time data. As will be shown below, D converged to similar values for architectures that successfully modeled the experimental data. The gain of the activation function ( $g$ in Eq. 1) was also optimized for architectures that included the locus coeruleus. The details of the gain optimization are presented below.

The Nelder-Mead simplex method was used to minimize the cost function (Nelder \& Mead, 1965), since it is effective at parameterizing connectionist models that have minimal noise (Bogacz \& Cohen, 2004). The Nelder-Mead is highly susceptible to getting stuck on a local minimum in the cost function and missing the global minimum. To reduce this problem, each optimization run started by randomly selecting the parameter values and computing the cost function. This process was repeated 1,000 times. The randomly selected parameter set that produced the lowest cost function was used as the starting values for that run's Nelder-Mead optimization. The upper and lower bounds of the parameter search in the Nelder-Mead were set to 0 and 10 for the excitatory connections, 0 and -10 for the inhibitory connections, and 300 to 560 for $\mathrm{D}$. These bounds restricted the parameter search space and thereby increased the probability of finding a parameter set that would produce an acceptable fit of the experimental data. The Nelder-Mead algorithm continued searching until a minimum cost function was found or until 10,000 iterations had been performed, whichever was least. Twenty optimization runs were computed for each of the model architectures. For two of the architectures (see below), an additional set of 20 optimization runs was performed to better characterize the parameter space that produced acceptable fits. In essentially all cases, the optimization run either provided an excellent fit or a clearly poor fit of the experimental data (see Table 2). Consequently, establishing strict acceptance criteria for the fits was not necessary. Acceptable fits were associated with cost function values that were less than $10^{-4}$.

\section{Results}

\section{Architectures with direct connections between the medial and lateral prefrontal cortices}

First we determined whether the architecture that produced the best qualitative fit of the reaction time and brain activation data in Dowman and ben-Avraham (2008) also produced a good quantitative fit. That architecture (labeled AtoS) is identical to that shown in Fig. 2, except that the sensory attention nodes (Av, As) have bidirectional excitatory connections with the early sensory nodes (Sv, Ss) instead of the late sensory nodes (Mv, Ms). The best fits of the 20 optimization runs for the AtoS architecture are shown in Table 2. Although the AtoS architecture produced an excellent quantitative fit of reaction times alone, it could not fit the reaction time and CTN data together (cost functions ranged from $1.7 \times 10^{-4}$ to $1.6 \times 10^{-2}$ ) it was unable to simulate the absence of a CTN cue validity effect in the pain-absent context. The AtoM architecture shown in Fig. 2 addressed this problem by having the sensory attention nodes connect to the late sensory nodes. This change was based on studies showing that voluntary attention has a larger effect on the later stages of sensory processing than on the earlier stages (Beck \& Kastner, 2009; Deco \& Rolls, 2005; Hsiao, O’Shaughnessy, \& Johnson 1993; Lavie, 2005; Poranen \& Hyvarinen, 1982). The AtoM architecture produced excellent quantitative fits of the both the reaction times and CTN amplitudes (Table 2).

Activation plots for the simulated CTN (Ss + Ths) and FCN (mPs) obtained from an AtoM optimization run that produced an acceptable fit are shown in Fig. 3. The polarity of the scalp potential depends on the orientation of the generator, which, owing to the convolutions of the cortex, will depend on its location (Nunez, 1981; Scherg, 1990). Consequently, the polarities of the simulated CTN and FCN activations were reversed for comparison with the experimental data shown in Fig. 1. Like the experimental data, the 
Table 2 Representative optimization run results for the model architectures

\begin{tabular}{|c|c|c|c|c|c|c|c|c|c|}
\hline \multirow[b]{3}{*}{ Architecture } & \multirow[b]{3}{*}{ Run \# } & \multirow{3}{*}{$\begin{array}{l}\text { \#Runs, } \\
\mathrm{J}(\mathrm{V})<10^{-4}\end{array}$} & \multirow[b]{3}{*}{$\mathrm{J}(\mathrm{V})$} & \multicolumn{4}{|c|}{ Reaction Time } & \multirow{3}{*}{$\begin{array}{l}\text { CTN } \\
\text { Pain Abs } \\
\% \uparrow I C(0 \%)\end{array}$} & \multirow{3}{*}{$\begin{array}{l}\text { Pain } \\
\% \uparrow \operatorname{IC~}(14.4 \%)\end{array}$} \\
\hline & & & & \multicolumn{2}{|l|}{ Pain Abs } & \multicolumn{2}{|l|}{ Pain } & & \\
\hline & & & & $\mathrm{VC}(684.5)$ & IC (809.8) & $\mathrm{VC}(566.4)$ & IC (616.2) & & \\
\hline AtoS & 15 & 9 & $5.3 \times 10^{-19}$ & 684.5 & 809.8 & 566.4 & 616.2 & - & - \\
\hline Atos & 11 & 0 & $1.7 \times 10^{-4}$ & 684.5 & 809.7 & 568.0 & 616.3 & -12.7 & 14.0 \\
\hline AtoM & 1 & 3 & $9.7 \times 10^{-15}$ & 684.5 & 809.8 & 566.4 & 616.2 & 0.0 & 14.4 \\
\hline mPstoA & 15 & 0 & $1.7 \times 10^{-2}$ & 616.7 & 812.7 & 616.5 & 643.4 & - & - \\
\hline $\mathrm{LCp}$ & 11 & 9 & $1.3 \times 10^{-16}$ & 684.5 & 809.8 & 566.4 & 616.2 & 0.0 & 14.4 \\
\hline $\mathrm{LCt}$ & 3 & 14 & $3.2 \times 10^{-16}$ & 684.5 & 809.8 & 566.4 & 616.2 & - & - \\
\hline
\end{tabular}

Abbreviations: Pain Abs = pain-absent context; Pain = pain context; $\mathrm{VC}=$ validly cued condition; $\mathrm{IC}=$ invalidly cued condition; $\% \uparrow \mathrm{IC}=$ percent increase in the invalidly cued condition; $\mathrm{J}(\mathrm{V})=$ cost function; Run $\#=$ the representative optimization run that produced an acceptable fit; \#Runs, $\mathrm{J}(\mathrm{V})<10^{-4}=$ the number of optimization runs that produced an acceptable fit of the experimental data; $-=$ data were not modeled. The reaction time and $\mathrm{CTN}$ values in parentheses are the experimental data

simulated CTN for the pain context was $14.4 \%$ larger in the invalidly cued than in the validly cued condition, and no cue validity effect emerged in the pain-absent context. As with our earlier modeling study, the FCN was larger in the invalidly cued than in the validly cued condition (Fig. 3); however, this fit can only be considered qualitative. (The P3a amplitude was not estimated from the response attention node [ARs] because the external inputs applied to this node contaminated the threat signal from the medial prefrontal cortex node [mPs]).

The simulated CTN amplitude for the AtoM architecture was larger in the pain context than in the pain-absent context (Fig. 3). This was due to activation of the threat detector (Ths) node in both the validly cued and invalidly cued conditions in the pain context, but not in the pain-absent context. The amplitudes of the somatosensory early sensory node (Ss) were the same across these conditions (data not shown). Hence, the architecture addressed the overall faster reaction times in the validly cued pain context (see Table 1) by adjusting the feedforward inhibitory input to the somatic threat detector node (Ths), so that there was some somatic threat detector activity in the validly cued condition. This finding contrasts with the experimental data, in which the CTN amplitudes obtained in the validly cued conditions of the pain-absent and pain contexts were comparable (Table 1). We ran another optimization for the AtoM architecture, with the additional constraint that the CTN amplitude be same in the pain and painabsent validly cued conditions (i.e., percent change in amplitude between the conditions $=0 \%$ ). None of the 20 optimization runs produced an acceptable fit (cost functions ranged from $2.0 \times 10^{-3}$ to $2.7 \times 10^{-2}$ ). Possible explanations for this discrepancy will be presented in the Discussion section.

Dowman and ben-Avraham (2008) reported that an architecture that directs the somatic threat signal from the medial prefrontal cortex (mPs) to the lateral prefrontal cortex areas involved in reorienting attention to sensory processes (As), instead of the areas reorienting attention to response processes (ARs), did not provide an acceptable qualitative fit of the reaction time data. We reexamined this architecture here to determine whether the optimization could find a parameter set that did provide an acceptable fit. As before, this architecture (labeled mPstoA) failed to fit the reaction time data (Table 2; cost functions ranged from $1.7 \times 10^{-2}$ to $5.7 \times 10^{-2}$ ).

\section{Locus coeruleus phasic response (LCp) architecture}

The model architectures examined so far have been based on the hypothesis that the somatic threat signal generated in the medial prefrontal cortex is sent to the lateral prefrontal cortex via direct anatomical connections. These connections have been demonstrated in a number of studies, and are a basic component of attentional control implemented in response conflict models (see Carter \& van Veen, 2007, for a review). An alternative architecture might involve the somatic threat signal in the medial prefrontal cortex eliciting a phasic response in the locus coeruleus and a subsequent phasic release of norepinephrine in the cerebral cortex. This phasic increase in norepinephrine would, in turn, increase the gain of the cortical neurons, and thereby facilitate responses that are timelocked to the evoking stimulus (Aston-Jones \& Cohen, 2005; Nieuwenhuis et al., 2005). We investigated the feasibility of this mechanism in the attentional bias toward somatic threats by using the architecture shown in Fig. 4 (labeled $L C p$ ). The early sensory $(\mathrm{S})$ to late sensory $(\mathrm{M})$ to response $(\mathrm{R})$ pathways, attention, somatic threat detector, and medial prefrontal cortex components are the same as in the AtoM architecture described above. However, instead of the medial prefrontal cortex ( $\mathrm{mPs}$ ) threat signal going to the lateral prefrontal cortex (ARs), it goes to the node simulating the locus coeruleus. This connection implements the anatomical connections between the medial prefrontal cortex and the locus coeruleus that have 


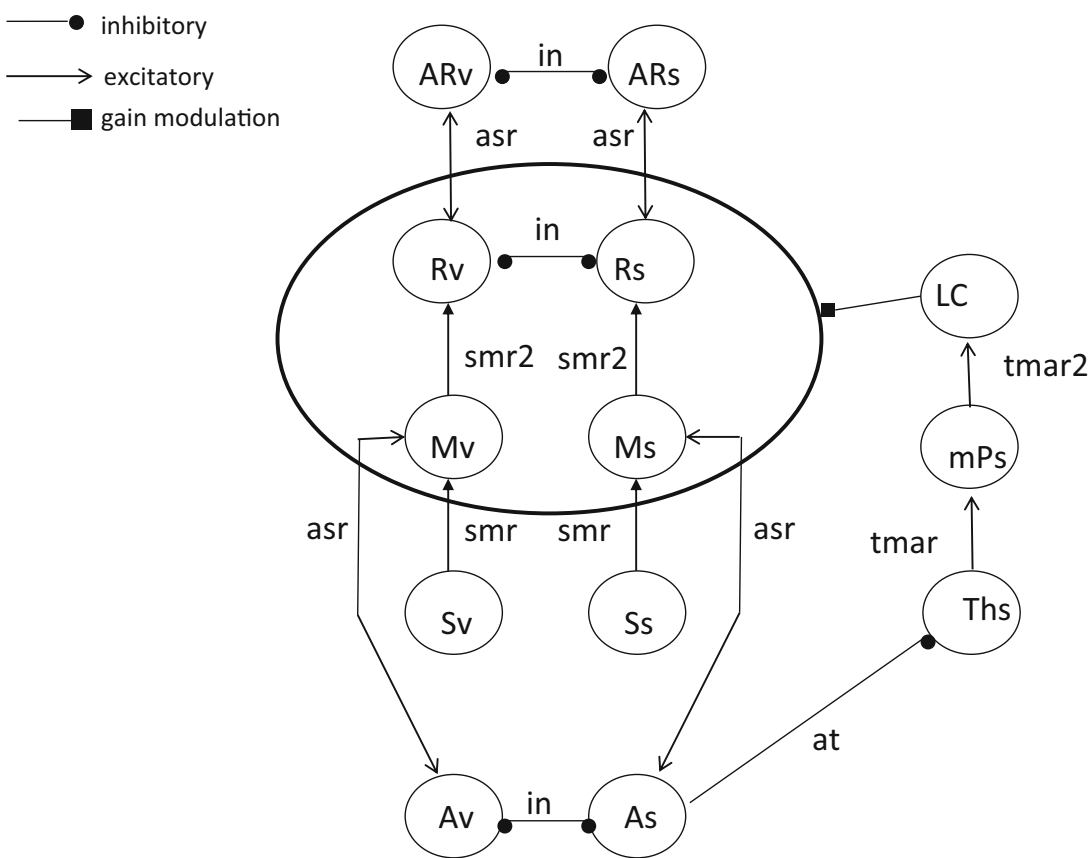

Fig. $4 \mathrm{LCp}$ architecture, simulating the phasic locus coeruleus hypothesis. LC is the node representing the locus coeruleus, the closed square represents gain that is modulated proportional to the LC activation value at each cycle, and the nodes whose gain are modulated are within the ellipse. All other abbreviations and symbols are the same as in Fig. 2. been shown in experimental work (Aston-Jones \& Cohen, 2005; Berridge \& Waterhouse, 2003; Sara \& Bouret, 2012). The timing of the locus coeruleus phasic response occurs too late to affect sensory processing, but rather, only affects decision and response processes (Aston-Jones \& Cohen, 2005; Nieuwenhuis et al., 2005). Hence, for the LCp architecture the changes in gain were limited to the response nodes $(\mathrm{Rv}$, Rs) and the late sensory nodes (Mv, Ms). For this architecture, the late sensory nodes are presumed to include categorization and stimulus-response mapping processes. Restricting the gain changes to the response nodes produced the same results.

Activation levels of the locus coeruleus node altered the gain of the late sensory and response node activation functions on each cycle according to

$g_{i}=1+g p^{*} A_{i}(\mathrm{LC})$,

where $g_{i}$ is the activation function gain (Eq. 1) on cycle $i, g p$ is the gain parameter, and $A_{i}(\mathrm{LC})$ is the activation level of the locus coeruleus node on cycle $i$. This provided a phasic change in gain that was proportional to the locus coeruleus node activation level. The $g p$ variable was optimized along with the connection strengths and the constant used to correct for perceptual, decision, and reaction times (D in Eq. 3), in order to find the best quantitative fit with both reaction times and CTN amplitudes. The Nelder-Mead optimization search bounds for $g p$ were set between 0 and 2 .
The LCp architecture produced excellent quantitative fits of reaction times and CTN amplitudes (Table 2). Activation plots for the simulated CTN (Ss + Ths) and FCN (mPs), and the locus coeruleus (LC), are shown in Fig. 5. The CTN amplitude obtained in the pain context was $14.4 \%$ larger in the invalidly cued than in the validly cued condition, and no cue validity effect was apparent in the pain-absent condition. The FCN and LC activation functions were greater in the invalidly cued than in the validly cued conditions, but as we noted above, these are only qualitative fits. As was the case for the AtoM architecture, the overall CTN amplitude was larger in the pain than in the pain-absent conditions. Adding the additional constraint that there be no change in the simulated CTN amplitude between the validly cued conditions in pain-absent and pain contexts did not result in any acceptable fits (cost functions ranged from $2.0 \times 10^{-3}$ to $2.9 \times 10^{-2}$ ).

\section{Locus coeruleus tonic response (LCt) architecture}

Finally, we examined whether the reaction time data could be accounted for by a simple change in general arousal. It is reasonable to assume that general arousal levels would be higher in a context that included painful sural-nerve stimuli than in one that did not. The increase in general arousal would be mediated in large part by an increase in the locus coeruleus tonic response, which in turn results in a tonic increase in the 


\section{LCp architecture activations}

\section{PAIN ABSENT}
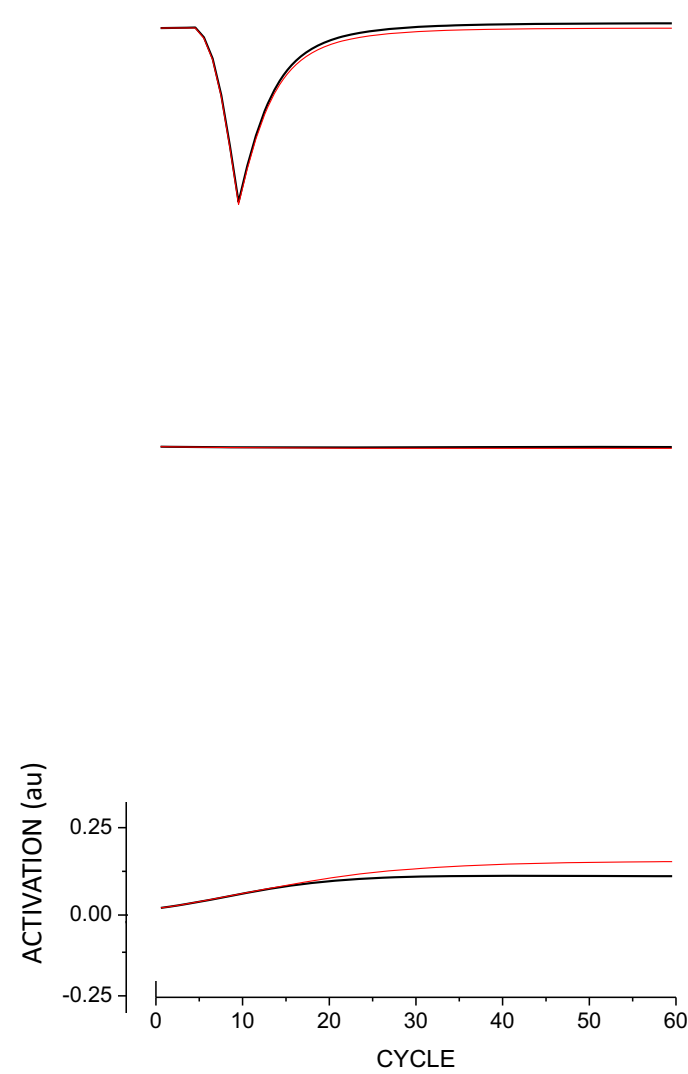

Fig. 5 Activation functions for the LCp architecture. Activation values are shown for nodes representing the CTN (combination of somatosensory sensory [Ss] and threat detector [Ths] nodes; upper panels), the FCN (somatosensory medial prefrontal cortex [mPs] nodes;

gain of the cortical neurons (Aston-Jones, 2005; Aston-Jones \& Cohen, 2005; Berridge \& Waterhouse, 2003; Sara, 2009). We simulated this by optimizing the activation function gain ( $g$ in Eq. 1) separately for the pain and pain-absent contexts, where the gain for the pain context was given by

$g_{p}=1+g p_{p}$

and the gain for the pain-absent context by

$g_{p a}=1+g p_{p a}$,

Note that $g_{p}$ and $g_{p a}$ were held constant across all cycles for a given optimization run. In each run, $g p_{p}$ and $g p_{p a}$, the connection strengths, and the constant used to correct reaction times were optimized to find the best quantitative fit of the reaction time data. Because the change in general arousal would affect the excitability of all cortical areas (AstonJones \& Cohen, 2005; Berridge \& Waterhouse, 2003; Sara,
PAIN

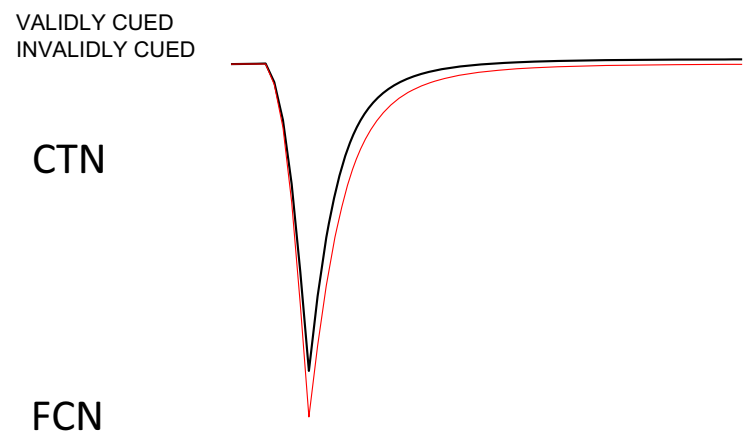

LC

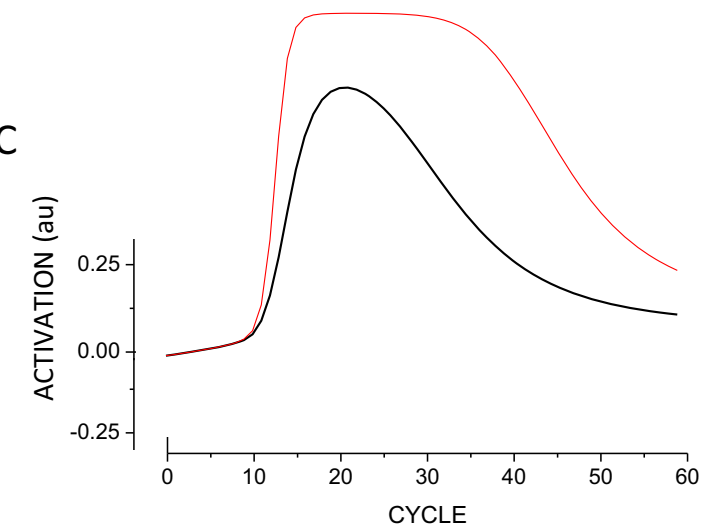

middle panels), and the locus coeruleus (LC; bottom panels), obtained in the different pain (pain-absent context, pain context) and cue validity (validly cued, invalidly cued) conditions. au = arbitrary units

2009), gains were modified for all nodes in the model (see the LCt architecture, Fig. 6).

The changes in general arousal produced excellent quantitative fits of the reaction time data (Table 2). The gain in the pain context was greater than the gain in the pain-absent context for each of the 14 optimization runs that produced an acceptable fit (the $M \pm S D g_{p a}$ and $g_{p}$ values were $1.691 \pm$ 0.304 and $2.590 \pm 0.418$, respectively). The higher gain is consistent with higher cortical arousal in the pain context. The LCt architecture optimization runs that produced an acceptable fit all exhibited early sensory attention node (Ss) activation levels that were quite high and that were larger in the pain than in the pain-absent context (Fig. 7).

\section{Preliminary analysis of the characteristics of model parameters producing acceptable fits}

Inspection of the successful optimization runs suggests that the parameter space producing acceptable fits of reaction 


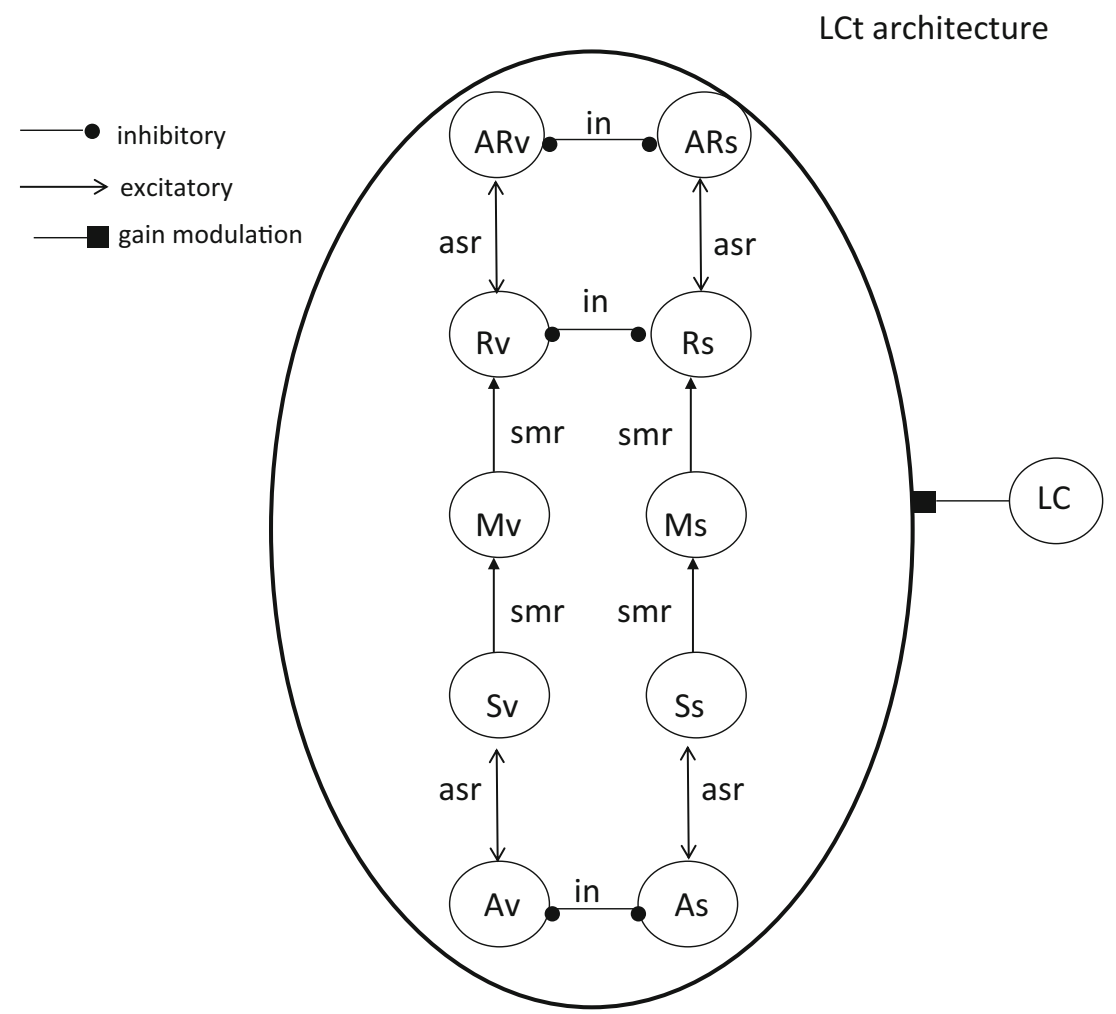

Fig. 6 Model architecture simulating the tonic locus coeruleus hypothesis (LCt), representing the effects of general arousal. All abbreviations and symbols are the same as in Figs. 2 and 4.

times and CTN amplitudes is quite narrow. An example is shown in Table 3A, which presents the nine acceptable bestfit parameters for the LCp architecture obtained over two sets

\section{LCt architecture Ss activation}

\section{Ss}

PAIN ABSENT

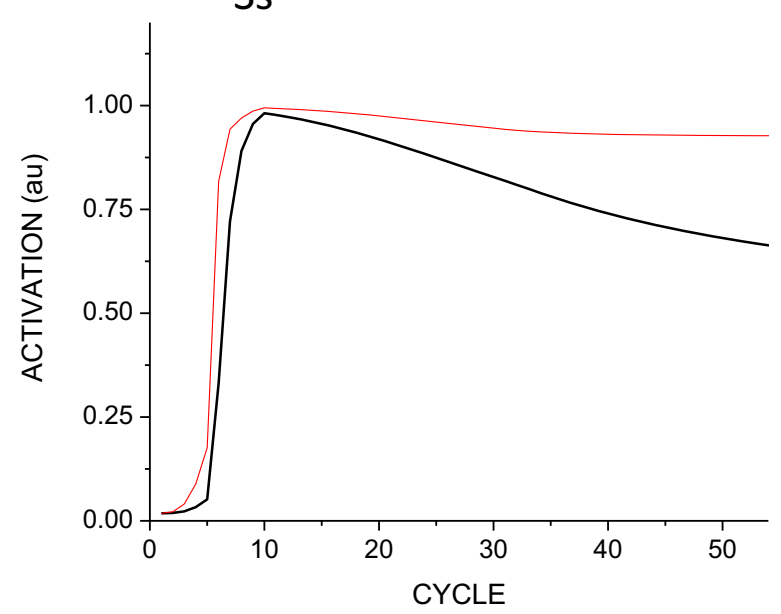

Fig. 7 Activation functions for the LCt architecture. The activations shown are for the node representing early sensory activity (Ss), obtained in the pain-absent and pain contexts and averaged across the cue validity conditions and the 14 optimization runs that produced an acceptable fit. (Cue validity had no effect on the Ss activation.) $a u=$ arbitrary units of 20 optimization runs. Note that the variability (measured by the $S D$ ) of the constant used to correct reaction times (D) and of the connection strength between the sensory attention and threat detector nodes (at) were low. Note also that some of the parameters appear to be linearly related, so that a change in one is compensated for by a change in another. The correlations between the model parameter values (Table 3B) demonstrate that this is the case. For example, the increase in the connection strength parameter connecting the threat detector and medial prefrontal cortex nodes (tmar) was offset by an increase in the constant used to correct reaction times (D; see Fig. 4). The increase in reaction times caused by the increase in $\mathrm{D}$ would have been compensated for by a decrease in reaction times resulting from an increase in tmar. Presumably holding D constant would have decreased the range of tmar values that produced an acceptable fit. The same applies to the other parameters showing significant correlations. Together, these data suggest that a narrow range of parameter values are necessary to produce an acceptable quantitative fit of the experimental data. Indeed, we found that to generate acceptable fits of the reaction time and CTN amplitude data by manually inserting fixed parameter values, it was necessary to use 16 decimal places; rounding to three decimal places did not produce an acceptable fit. Similar results were obtained for the other architectures that produced acceptable fits of the reaction time and CTN data, though the interdependencies between parameter values depended on the architecture (see 
Table 3 LCp architecture

A. Optimized parameter values producing acceptable fits with the reaction time and CTN data Model Parameters

\begin{tabular}{|c|c|c|c|c|c|c|c|c|c|c|c|}
\hline Set & Run \# & Cost & $\mathrm{D}$ & asr & at & in & smr & smr2 & tmar & tmar2 & gp \\
\hline 1 & 5 & $1.8 \times 10^{-14}$ & 381.7 & 0.793 & -0.153 & -3.306 & 6.977 & 0.265 & 9.807 & 1.391 & 1.358 \\
\hline 1 & 6 & $1.2 \times 10^{-13}$ & 352.1 & 0.830 & -0.156 & -8.289 & 7.794 & 0.309 & 4.415 & 1.923 & 1.302 \\
\hline 1 & 11 & $1.3 \times 10^{-16}$ & 300.6 & 0.757 & -0.153 & -4.528 & 9.990 & 0.197 & 1.394 & 9.912 & 1.148 \\
\hline 1 & 16 & $2.9 \times 10^{-14}$ & 364.1 & 0.786 & -0.158 & -8.684 & 5.165 & 0.342 & 6.002 & 1.553 & 1.639 \\
\hline 1 & 17 & $2.5 \times 10^{-14}$ & 358.6 & 0.077 & -0.164 & -9.968 & 0.986 & 3.121 & 9.501 & 9.052 & 0.374 \\
\hline 2 & 3 & $2.3 \times 10^{-14}$ & 300.4 & 0.823 & -0.157 & -9.983 & 7.783 & 0.267 & 3.567 & 1.880 & 1.105 \\
\hline 2 & 4 & $7.4 \times 10^{-14}$ & 313.1 & 0.558 & -0.160 & -8.448 & 3.979 & 0.328 & 3.219 & 5.232 & 0.686 \\
\hline 2 & 8 & $2.3 \times 10^{-13}$ & 352.3 & 0.581 & -0.158 & -6.248 & 3.786 & 0.351 & 9.726 & 1.612 & 0.927 \\
\hline \multirow[t]{3}{*}{2} & 16 & $1.7 \times 10^{-14}$ & 329.7 & 0.768 & -0.153 & -4.116 & 9.301 & 0.233 & 8.697 & 0.748 & 1.778 \\
\hline & $M$ & $6.4 \times 10^{-14}$ & 339.2 & 0.663 & -0.157 & -7.063 & 6.196 & 0.601 & 6.259 & 3.700 & 1.146 \\
\hline & $S D$ & $8.5 \times 10^{-14}$ & 29.4 & 0.242 & 0.004 & 2.572 & 2.930 & 0.946 & 3.254 & 3.519 & 0.443 \\
\hline
\end{tabular}

B. Acceptable fit optimized parameter Pearson correlations

\begin{tabular}{|c|c|c|c|c|c|c|c|c|}
\hline & asr & at & in & smr & $\mathrm{smr} 2$ & tmar & tmar2 & $g p$ \\
\hline D & -0.173 & -0.121 & 0.135 & -0.413 & 0.271 & 0.755 & -0.337 & 0.125 \\
\hline asr & & 0.822 & 0.392 & 0.824 & -0.920 & -0.378 & -0.589 & 0.810 \\
\hline at & & & 0.804 & 0.912 & -0.753 & -0.147 & -0.345 & 0.769 \\
\hline in & & & & 0.548 & -0.450 & 0.244 & -0.138 & 0.493 \\
\hline $\mathrm{smr}$ & & & & & -0.703 & -0.440 & -0.208 & 0.711 \\
\hline $\mathrm{smr} 2$ & & & & & & 0.386 & 0.549 & -0.665 \\
\hline tmar & & & & & & & -0.343 & 0.010 \\
\hline tmar2 & & & & & & & & -0.640 \\
\hline
\end{tabular}

The statistically significant $(p<.05)$ correlation values are given in bold

Abbreviations and symbols: Set $=$ set of 20 optimization runs; Run \# = the optimization run producing an acceptable fit. Runs not producing an acceptable fit are not shown. Cost $=$ cost function; $\mathrm{D}=$ constant used to account for the perceptual, decision, and response selection processes not included in the model; asr, at, in, smr, smr2, tmar, and tmar2 are the connection strengths between nodes (see Fig. 4), and $g p$ is the gain parameter used to optimize the activation gain

Tables 4 and 5). (For a more thorough analysis of the parameter space, see Ritz, Fowler, \& Dowman, manuscript in preparation).

\section{Discussion}

To our knowledge, this study is the first attempt at using connectionist modeling to quantitatively fit reaction time and ERP data to investigate the brain mechanisms underlying the ability of a biologically significant stimulus to reorient attention. The results confirm the feasibility of the somatic threat-detectionand-reorienting hypothesis proposed by Dowman and benAvraham (2008), and have revealed two other physiologically plausible mechanisms. Importantly, not all of the model architectures examined here could produce an acceptable fit of the experimental data. We cannot, of course, exclude the possibility that for these architectures the Nelder-Mead optimization merely failed to find a parameter set that produced an acceptable fit of the experimental data. We feel this is unlikely, however, given the extensive preliminary work we performed developing the optimization - that is, choosing the best fit of 1,000 random iterations for the starting model parameters, determining the model parameter bounds, allowing up to 10,000 iterations to find the global minimum, and performing 20 optimization runs. In our experience, the architectures that failed to produce an acceptable fit of the experimental data continued to do so even when we increased these optimization parameters. Future work will be necessary to determine whether other optimization techniques, such as the genetic algorithm, can find an acceptable fit. Nonetheless, our results suggest that merely varying a large number of model parameters does not always produce a successful fit. Rather, the model architecture is important. Indeed, as we argue below, the architectures that produced acceptable quantitative fits of the experimental data are more closely aligned with the empirical findings than are those that did not.

\section{Comparison of model architectures with empirical data}

The somatic threat-detection-and-reorienting hypothesis was a modification of the response conflict model (e.g., Botvinick 
Table 4 AtoM architecture

A. Optimized parameter values producing acceptable fits with the reaction time and CTN data Model Parameters

\begin{tabular}{|c|c|c|c|c|c|c|c|c|c|c|}
\hline Set & Run \# & Cost & $\mathrm{D}$ & asr & at & in & smr & smr2 & tmar & tmar2 \\
\hline 1 & 1 & $9.71 \times 10^{-15}$ & 362.8 & 0.419 & -0.157 & -3.556 & 1.805 & 0.509 & 5.742 & 6.309 \\
\hline 1 & 4 & $2.81 \times 10^{-14}$ & 358.7 & 0.465 & -0.162 & -9.974 & 1.888 & 0.537 & 7.430 & 4.437 \\
\hline 1 & 14 & $3.17 \times 10^{-14}$ & 381.4 & 0.605 & -0.160 & -8.680 & 1.143 & 0.619 & 8.737 & 6.146 \\
\hline 2 & 8 & $1.54 \times 10^{-14}$ & 351.6 & 0.328 & -0.157 & -2.525 & 2.064 & 0.491 & 5.479 & 6.838 \\
\hline 2 & 19 & $4.79 \times 10^{-15}$ & 367.4 & 0.436 & -0.156 & -2.596 & 1.620 & 0.495 & 5.540 & 7.123 \\
\hline \multirow[t]{3}{*}{2} & 20 & $1.22 \times 10^{-14}$ & 364.7 & 0.514 & -0.161 & -9.473 & 1.687 & 0.544 & 6.588 & 5.767 \\
\hline & $M$ & $1.70 \times 10^{-14}$ & 364.4 & 0.461 & -0.159 & -6.134 & 1.701 & 0.533 & 6.586 & 6.103 \\
\hline & $S D$ & $1.07 \times 10^{-14}$ & 10.0 & 0.093 & 0.002 & 3.593 & 0.315 & 0.048 & 1.294 & 0.950 \\
\hline
\end{tabular}

B. Acceptable fit optimized parameter Pearson correlations

\begin{tabular}{|c|c|c|c|c|c|c|c|}
\hline & asr & at & in & smr & smr2 & tmar & tmar2 \\
\hline D & 0.888 & -0.164 & -0.347 & -0.995 & 0.803 & 0.684 & 0.073 \\
\hline asr & & -0.592 & -0.736 & -0.890 & 0.924 & 0.864 & -0.338 \\
\hline at & & & 0.980 & 0.183 & -0.619 & -0.730 & 0.896 \\
\hline in & & & & 0.361 & -0.724 & -0.800 & 0.832 \\
\hline smr & & & & & -0.829 & -0.711 & -0.084 \\
\hline smr2 & & & & & & 0.958 & -0.359 \\
\hline tmar & & & & & & & -0.548 \\
\hline
\end{tabular}

The statistically significant $(p<.05)$ correlation values are given in bold

Abbreviations and symbols: Set $=$ set of 20 optimization runs; Run \# = the optimization run producing an acceptable fit. Runs not producing an acceptable fit are not shown. Cost = cost function; $\mathrm{D}=$ constant used to account for the perceptual, decision, and response selection processes not included in the model; asr, at, in, smr, smr2, tmar, and tmar2 are the connection strengths between nodes (see Fig. 2)

et al., 2001; Carter \& van Veen, 2007; Yeung et al., 2004), in which the response conflict component was replaced by a threat detector (see Dowman \& ben-Avraham, 2008). This hypothesis proposed that somatic threats are detected by somatic threat detectors in the dorsal posterior insula. Activation of the somatic threat detectors is monitored by the medial prefrontal cortex, which in turn signals the lateral prefrontal cortex to redirect attention toward the threat. The flow of information between these brain areas is assumed to involve direct anatomical connections, as is the case for the response conflict models. The AtoM architecture testing this hypothesis (Fig. 2) was able to produce excellent quantitative fits of the reaction time and the somatic threat detector activity indexed by the CTN. To quantitatively fit the reaction times and CTN amplitudes, voluntary attention initiated by the cue was directed toward late sensory processes (the M node in Fig. 2). Directing voluntary attention toward the early sensory stage (S in Fig. 2) could not fit the absence of a cue validity effect on CTN amplitudes that we observed in the pain-absent context. This is consistent with studies demonstrating that the effects of voluntary attention are greater in the higher-order sensory cortices than in the early sensory cortices (Beck \& Kastner, 2009; Deco \& Rolls, 2005; Hsiao et al., 1993; Lavie, 2005; Poranen \& Hyvarinen, 1982).

The architecture that sent the threat signal from the medial prefrontal cortex node to the sensory attention node (mPstoA) could not generate an acceptable fit of the reaction time and CTN data. This result suggests that the effect of somatic threat on reaction times is due to attention being redirected toward decision and/or response processes, and not to sensory processes. This finding fits with our observation that sural-nerve intensity ratings are lower in the invalidly cued than in the validly cued condition, whereas the CTN amplitude presumed to index the somatic threat detectors is greater in the invalidly cued condition (Dowman, 2001, 2007a). These data do not mean that the threat signal elicited in the medial prefrontal cortex is not sent to the lateral prefrontal cortex areas controlling attention to the sensory areas. It might be the case that the signal reaches the sensory areas too late to produce any effect on sensory processing. Other studies investigating the effects of salient stimuli on sensory processing have reported similar effects. For example, Prinzmetal, McCool, and Park (2005) showed that a predictive salient cue enhanced sensory processing of a target stimulus at a cue-target stimulus onset asynchrony (SOA) of $300 \mathrm{~ms}$, but not at SOAs of 0 or $150 \mathrm{~ms}$. This result suggests that the cueevoked orienting of attention toward sensory processes takes somewhere around $300 \mathrm{~ms}$. It is unlikely, therefore, that the reorienting of attention elicited by a biologically significant stimulus such as pain would affect its own sensory processing.

One observation that cannot be addressed by the threatdetection-and-reorienting architecture is the latency between 
Table 5 LCt architecture

A. Optimized parameter values producing acceptable fits with the reaction time and $\mathrm{CTN}$ data

\begin{tabular}{clllllll}
\multicolumn{7}{c}{ Model Parameters } \\
Run \# & Cost & D & asr & in & smr & $g p_{p a}$ & $g p_{p}$ \\
2 & $2.74 \times 10^{-13}$ & 372.6 & 0.362 & -1.927 & 0.310 & 0.914 & 1.638 \\
3 & $3.25 \times 10^{-16}$ & 462.5 & 0.370 & -6.365 & 0.701 & 0.485 & 1.753 \\
5 & $7.75 \times 10^{-14}$ & 379.3 & 0.358 & -5.523 & 0.381 & 0.891 & 1.731 \\
7 & $2.47 \times 10^{-05}$ & 485.0 & 0.391 & -7.531 & 1.024 & 0.257 & 1.536 \\
9 & $1.57 \times 10^{-14}$ & 313.6 & 0.331 & -8.155 & 0.320 & 0.942 & 1.624 \\
10 & $8.38 \times 10^{-15}$ & 358.0 & 0.354 & -6.935 & 0.361 & 0.919 & 1.694 \\
11 & $6.99 \times 10^{-07}$ & 484.0 & 0.375 & -8.044 & 1.010 & 0.266 & 1.632 \\
12 & $7.46 \times 10^{-14}$ & 372.4 & 0.357 & -3.323 & 0.347 & 0.910 & 1.691 \\
13 & $3.18 \times 10^{-14}$ & 406.8 & 0.360 & -9.972 & 0.459 & 0.815 & 1.837 \\
14 & $4.72 \times 10^{-15}$ & 446.6 & 0.385 & -2.345 & 0.492 & 0.670 & 1.786 \\
15 & $1.00 \times 10^{-13}$ & 377.3 & 0.356 & -4.198 & 0.366 & 0.899 & 1.714 \\
16 & $5.35 \times 10^{-16}$ & 303.5 & 0.356 & -0.187 & 0.447 & 0.042 & 0.167 \\
17 & $1.21 \times 10^{-13}$ & 363.0 & 0.358 & -2.517 & 0.322 & 0.910 & 1.627 \\
20 & $2.22 \times 10^{-14}$ & 419.8 & 0.359 & -9.302 & 0.494 & 0.761 & 1.828 \\
$M$ & $1.82 \times 10^{-06}$ & 396.0 & 0.362 & -5.452 & 0.502 & 0.691 & 1.590 \\
$S D$ & $6.60 \times 10^{-06}$ & 57.5 & 0.015 & 3.053 & 0.241 & 0.304 & 0.418
\end{tabular}

B. Acceptable fit optimized parameter Pearson correlations

$\begin{array}{llllll} & \text { asr } & \text { in } & \mathrm{smr} & \mathrm{gp} & \mathrm{gp} \\ \mathrm{D} & \mathbf{0 . 8 4 1} & -0.402 & \mathbf{0 . 8 1 8} & -0.361 & 0.459 \\ \text { asr } & & 0.044 & \mathbf{0 . 7 2 4} & \mathbf{- 0 . 5 2 9} & 0.104 \\ \text { in } & & & -0.384 & -0.083 & \mathbf{- 0 . 5 2 2} \\ \text { smr } & & & & \mathbf{- 0 . 7 3 6} & 0.002 \\ \text { gppa } & & & & & \mathbf{0 . 6 4 6}\end{array}$

The statistically significant $(p<.05)$ correlation values are given in bold

Abbreviations and symbols: Set $=$ set of 20 optimization runs; Run $\#=$ the optimization run producing an acceptable fit. Runs not producing an acceptable fit are not shown. Cost $=$ cost function; $\mathrm{D}=$ constant used to account for the perceptual, decision, and response selection processes not included in the model; asr, in, and smr are the connection strengths between nodes, and $g p_{p a}$ and $g p_{p}$ are the gain parameters for the pain-absent and pain contexts, respectively, used to optimize the activation gain (see Fig. 6)

the threat signal elicited in the medial prefrontal cortex (indexed by the FCN) and the subsequent reorienting of attention toward the decision and response processes presumed to be mediated by the lateral prefrontal cortex (indexed by the P3a). The grand average peak latencies for the FCN and $\mathrm{P} 3 \mathrm{a}$ are about 150 and $360 \mathrm{~ms}$, respectively (Dowman, 2007a, b, 2014). Our connectionist models were not designed to realistically incorporate the timing of responses between the brain areas. Nonetheless, one would expect that transmission of the threat signal from the medial to the lateral prefrontal cortex via a direct synaptic connection would occur much faster than the observed $210 \mathrm{~ms}$, given that intracortical transmission times occur on the order of tens of milliseconds (e.g., Perez \& Cohen, 2009).

The phasic locus coeruleus architecture ( $\mathrm{LCp}$ ) provides a much more reasonable accounting of this latency difference.
The two major sources of cortical input to the locus coeruleus are the orbitofrontal cortex and the medial prefrontal cortex (Aston-Jones \& Cohen, 2005; Nieuwenhuis et al., 2005; Sara \& Bouret, 2012). Hence, the somatic threat signal generated in the medial prefrontal cortex will elicit a phasic response in the locus coeruleus via a direct anatomical connection. The timing of the threat signal in the medial prefrontal cortex $(\sim 150 \mathrm{~ms})$ suggests that the threat-evoked phasic response in the locus coeruleus would follow closely thereafter. However, there would be a considerable delay in the resulting phasic release of norepinephrine in the cerebral cortex, owing to the locus coeruleus efferent axons having very slow conduction velocities $(<1 \mathrm{~m} / \mathrm{s}$; Aston-Jones \& Cohen, 2005; Nieuwenhuis et al., 2005). The timing of this locus-coeruleus-mediated facilitation of cortical activity has been shown to coincide with the P300 ERP, which includes the P3a component of interest here (Nieuwenhuis et al., 2005). Hence, the phasic locus coeruleus architecture provides a much better account of the timing between the validity effects in the FCN and P3a reported in our experimental studies than does the architecture based on direct anatomical connections between the medial and lateral prefrontal cortices.

The phasic locus coeruleus architecture does not require that the reorienting of attention toward the decision and response processes elicited by the invalidly cued sural-nerve target be mediated by the lateral prefrontal cortex, but rather could do so by directly facilitating the neurons involved in these processes (Aston-Jones \& Cohen, 2005). In this case, the P3a validity effect might index some other process. For example, the change in attentional control that is initiated by response conflict is implemented on the following trial, not the current one (Brown, Reynolds, \& Braver, 2007; Carter \& van Veen, 2007; Walsh, Buonocore, Cameron, \& Mangun, 2011; Yeung et al., 2004). The same may be true for the processes indexed by the P3a validity effect observed in our studies. This would be consistent with the idea that the P3a generators play some role in updating the working memory template (Escera \& Corral, 2007; Friedman et al., 2001; Polich, 2003). Alternatively, the P3a may reflect inhibition of the visual discrimination task set (Polich, 2007; Sara \& Bouret, 2012; Wessel \& Aron, 2015). This might explain why we did not see a consistent relationship between P3a amplitude and attention reorienting in our experiments. Clearly, more work will be needed to determine the role of the P3a generators in the reorienting of attention toward unexpected biologically significant stimuli such as pain.

The tonic locus coeruleus architecture (LCt) simulating the effects of general arousal also provided excellent quantitative fits of the faster reaction times in the pain than in the painabsent contexts, including this difference being greater in the invalidly than in the validly cued condition. The latter effect arises from the nonlinear activation function of the neurons simulated by the nodes (see Deco \& Rolls, 2005, for other 
examples). In this case, the cue validly effects evident for the $\mathrm{CTN}, \mathrm{FCN}$, and P3a may not be related to reorienting attention to the potential somatic threat. Instead, they may be involved in detecting a stimulus that does not match the expected (i.e., cued) input. The cross-modal cuing paradigm used in our experimental studies confounds attention and expectation, where in the invalidly cued condition attention is directed elsewhere and the target stimulus is unexpected. Both attention and expectancy can affect sensory response amplitudes, but by different mechanisms: Attention facilitates the response via an excitatory bias on the sensory neurons (D'Esposito, 2007; Kastner \& Ungerleider, 2000; Seidl, Peelen, \& Kastner, 2012; Sylvester, Shulman, Jack, \& Corbetta, 2009). Expectation, on the other hand, involves a reduction in the sensory response as the expectancy develops, as with regular presentation of the stimulus and/or by instruction. When the stimulus is unexpected, this suppression is removed, resulting in greater sensory response amplitudes in the unexpected than in the expected condition (Summerfield \& Egner, 2009).

Recent experimental and computational modeling work suggests that the expectation effect is mediated by two classes of sensory neurons: representational neurons and prediction error neurons (Egner, Monti, \& Summerfield 2010; Summerfield \& Egner, 2009; Wacongne, Changeux, \& Dehaene, 2012). The representational neurons receive topdown input from higher-order sensory areas coding the stimulus expected on the current trial. A mismatch between representational-neuron activity and the sensory input will result in activation of the prediction error neurons, whose output is then used to update stimulus expectancy (Summerfield \& Egner, 2009). This raises the possibility that the somatic threat detectors described here are actually somatic threat prediction error neurons. That is, the larger CTN amplitude in the invalidly cued condition may reflect the activation of prediction error neurons signaling the occurrence of an unexpected (i.e., invalidly cued) potentially threatening somatic stimulus, and the FCN and P3a generators may be involved in updating the working memory template to incorporate the deviant stimulus (Friedman et al., 2001; Polich, 2003).

\section{Study limitations}

As is the case with many connectionist modeling studies (e.g., Botvinick et al., 2001; Yeung et al., 2004), our model did not attempt to incorporate details of the sensory, decision, and response processes associated with the color and sural-nerve intensity discrimination tasks, nor did we incorporate details of the processes that are responsible for the larger CTN in the invalidly cued condition. Rather, we chose a coarse-grained modeling approach that focuses on the overall magnitude and sequence of activity in the brain areas thought to be involved in these processes. This approach fits well with the nature of the ERP potentials used in modeling, which likewise give an estimate of the overall magnitude of generator activity, and not details of the microstructure. This coarse-grained modeling approach is acceptable as long as it captures the essence of the processes being modeled, which appears to be the case here. Nonetheless, it would be useful in future studies to more realistically model the activation functions and timing of the responses between connected nodes, to more accurately reflect the response properties of the brain areas being simulated.

As we noted in the Method section, questions about the overlap between the multiple, functionally diverse generator sources for the FCN and P3a components prevented us from using them in the modeling studies. This is an important issue, since it could help address questions raised here about whether the validity effect observed in these components is related to reorienting attention toward the unexpected target, updating stimulus expectancy, or interrupting an invalidly cued task set. Clearly, more work will be necessary to better isolate the activity related to reorienting attention, perhaps by using methods such as independent component analysis (e.g., Makeig, Debener, Onton, \& Delorme, 2004).

Although the nonpainful sural-nerve stimulus currents used in the pain and pain-absent contexts were comparable, the small but statistically significant differences between them may have had important consequences that we had not anticipated. Both stimulus currents produced comparable nonpainful paresthesia sensations; however, the stimulus current was higher in the pain-absent than in the pain context $(1.56 \pm 0.67$ vs. $0.95 \pm 0.28 \mathrm{~mA}$, respectively), $t(40)=3.39$, $p=.002$. CTN amplitudes increase with increasing nonpainful stimulus intensity (Dowman, 1994), so it is possible that the equivalence between the CTN amplitudes for the pain and pain-absent contexts observed in the experimental studies was due to the larger stimulus current in the pain-absent context producing an increase in CTN amplitudes that matched the increase due to the addition of somatic threat detector activity in the pain context predicted by the phasic locus coeruleus architecture. We are currently reexamining this question using more precise control of stimulus intensity, to make sure that the stimulus currents are indeed equivalent in the pain and pain-absent contexts.

\section{Conclusions and predictions derived from modeling}

The connectionist modeling study presented here revealed two physiologically feasible mechanisms underlying the faster reaction times for potential somatic threats: One involves somatic threat detectors eliciting activation of the medial prefrontal cortex, which in turn produces a phasic response in the locus coeruleus (LCp architecture). The other involves a tonic increase in locus coeruleus activity arising from the presence of pain ( $\mathrm{LCt}$ architecture). These two mechanisms provide competing predictions that can be tested in future studies, 
and thereby can provide valuable insight into which of them was responsible for the attentional bias toward potentially threatening somatic targets reported in our experimental studies. These predictions are detailed below.

1. The phasic locus coeruleus architecture predicts that the presence of pain will only affect sural-nerve target reaction times and not the visual-task reaction times, whereas the tonic locus coeruleus architecture predicts that both the sural-nerve and visual-target reaction times will be affected. If the faster invalidly cued reaction time for the sural-nerve target in the pain context is due to the locus coeruleus phasic response, then the resulting increase in excitability should be restricted to cortical areas involved in the decision (e.g., in the stimulus-response mapping) and/or response processes elicited by the sural-nerve target. Since this phasic change in excitability has a relatively short duration (about $100 \mathrm{~ms}$; see Nieuwenhuis et al., 2005; Sara \& Bouret, 2012), it should not have any effects on the visual-task reaction times. If, on the other hand, the reaction time effects are due to the locus coeruleus tonic response, then a general increase in cortical excitability should result in an increase in the sensory, decision, and response processes elicited by both the sural-nerve and visual targets. In this case, both the sural-nerve and visual-target reaction times would be faster in the pain than in the pain-absent context. Unfortunately, we cannot test this hypothesis with existing data, since a minor difference in the visual tasks of the two studies might have affected their reaction times: In Dowman (2007b, painabsent context), the participants were instructed to focus their gaze on the cued target, whereas in Dowman (2014, pain context), the participants maintained their gaze on a point in between their ankle and the LEDs and shifted their attention covertly. We are currently investigating this prediction by using exactly the same visual task in both the pain and pain-absent conditions.

2. The phasic locus coeruleus architecture predicts that the sural-nerve CTN and not the other sensory components of the sural-nerve and visual ERPs will be larger in the pain than in the pain-absent context, whereas the tonic locus coeruleus architecture predicts that all of the sensory components of the sural-nerve and visual ERPs will be larger in the pain context. The phasic locus coeruleus architecture accounted for the overall faster sural-nerve target reaction times in the pain than in the pain-absent contexts by having some somatic threat detector activity present in the validly cued condition. If this is the case, then the CTN should be larger in the pain context (composed of sensory and somatic threat detector activity) than in the painabsent context (sensory activity only). The tonic locus coeruleus architecture also predicts that the CTN will be larger in the pain than in the pain-absent context, albeit for a different reason. The tonic locus coeruleus architecture predicts that in the pain context there will be a tonic increase in the excitability of widespread regions of the cerebral cortex, including those areas involved in tactile and nociceptive somatic and visual sensory processing. Since this increase in excitability would be in place when the target stimulus was presented, both the visual and sural-nerve target stimuli should evoke greater activity in the sensory cortices (Berridge \& Waterhouse, 2003). For the sural-nerve target, this means not only a larger CTN amplitude, but also an increase in the amplitude of a negative potential that occurs at about $80 \mathrm{~ms}$ poststimulus and is generated in the primary somatosensory cortex (which we have labeled the central negativity or $C N$; Dowman, 2007a, 2014; Dowman \& Darcey, 1994; Dowman et al., 2007; Dowman \& Schell, 1999). Hence, if the tonic locus coeruleus architecture is correct, then both the CN and CTN of the sural-nerve ERP and the early sensory components of the visual ERP should be larger in the pain than in the pain-absent context. If, on the other hand, the phasic locus coeruleus architecture is correct, only the CTN amplitude would be larger in the pain context.

3. The phasic locus coeruleus architecture predicts that only the sural-nerve target would elicit a phasic pupil dilation in the pain context, whereas the tonic locus coeruleus architecture predicts that the tonic pupil diameter would be larger in the pain than in the pain-absent context. A number of studies have shown that pupil diameter closely follows the tonic and phasic locus coeruleus discharge rates (Aston-Jones \& Cohen, 2005; Gilzenrat, Nieuwenhuis, Jepma, \& Cohen, 2010; Nieuwenhuis et al., 2005; Nieuwenhuis, De Geus, \& Aston-Jones, 2011). Hence, measuring pupil diameters will help determine the roles of tonic and phasic locus coeruleus activity in the attentional bias toward potential somatic threats.

Author note This research work was supported by National Science Foundation UBM (Undergraduate Biology-Mathematics) Grant No. DBI-0926568. We also thank Keegan Lowenstein and Brian Leventhal for their expert help in the early stages of developing the connectionist modeling software.

\section{References}

Aston-Jones, G. (2005). Brain structures and receptors involved in alertness. Sleep Medicine, 6(Suppl. 1), S3-S7.

Aston-Jones, G., \& Cohen, J. D. (2005). An integrative theory of locus coeruleus-norepinephrine function: Adaptive gain and optimal performance. Annual Review of Neuroscience, 28, 403-450. doi:10. 1146/annurev.neuro.28.061604.135709

Basbaum, A., \& Jessel, T. M. (2000). The perception of pain. In E. R. Kandel, J. H. Schwartz, \& T. M. Jessel (Eds.), Principles of neural science (pp. 472-491). New York, NY: McGraw-Hill. 
Beck, D. M., \& Kastner, S. (2009). Top-down and bottom-up mechanisms in biasing competition in the human brain. Vision Research, 49, 1154-1165. doi:10.1016/j.visres.2008.07.012

Berridge, C. W., \& Waterhouse, B. D. (2003). The locus coeruleusnoradrenergic system: Modulation of behavioral state and statedependent cognitive processes. Brain Research Reviews, 42, 33-84.

Bishop, S. J. (2008). Neural mechanisms underlying selective attention to threat. Annuals of the New York Academy of Sciences, 1129, 141152

Bogacz, R., \& Cohen, J. D. (2004). Parameterization of connectionist models. Behavior Research Methods, Instruments, \& Computers, 36, 732-741. doi:10.3758/BF03206554

Botvinick, M. M., Braver, T. S., Barch, D. M., Carter, C. S., \& Cohen, J. D. (2001). Conflict monitoring and cognitive control. Psychological Review, 108, 624-652. doi:10.1037/0033-295X.108.3.624

Brown, J. W., Reynolds, J. R., \& Braver, T. S. (2007). A computational model of fractionated conflict-control mechanisms in taskswitching. Cognitive Psychology, 55, 37-85.

Bushnell, M. C., Duncan, G. H., Dubner, R., Jones, R. L., \& Maixner, W. (1985). Attentional influences on noxious and innocuous cutaneous heat detection in humans and monkeys. Journal of Neuroscience, 5 , $1103-1110$

Carter, C. S., \& van Veen, V. (2007). Anterior cingulate cortex and conflict detection: An update of theory and data. Cognitive, Affective, \& Behavioral Neuroscience, 7, 367-379. doi:10.3758/CABN.7.4.367

Cohen, J. D., Dunbar, K., \& McClelland, J. L. (1990). On the control of automatic processes: A parallel distributed processing account of the Stroop effect. Psychological Review, 97, 332-361. doi:10.1037/ 0033-295X.97.3.332

Cole, M. W., Yarkoni, T., Repovs, G., Anticevic, A., \& Braver, T. S. (2012). Global connectivity of prefrontal cortex predicts cognitive control and intelligence. Journal of Neuroscience, 32, 8988-8999. doi:10.1523/JNEUROSCI.0536-12.2012

Corbetta, M., Patel, G., \& Shulman, G. L. (2008). The reorienting system of the human brain: From environment to theory of mind. Neuron, $58,306-324$

Corbetta, M., \& Shulman, G. L. (2002). Control of goal-directed and stimulus-driven attention in the brain. Nature Reviews Neuroscience, 31, 201-215.

Craig, A. D. (2002). How do you feel? Interoception: The sense of the physiological condition of the body. Nature Reviews Neuroscience, 3, 655-666. doi:10.1038/nrn894

Craig, A. D. (2003). Interoception: The sense of the physiological condition of the body. Current Opinion in Neurobiology, 13, 500-505.

Crombez, C., Eccleston, C., Baeyens, F., \& Eelen, P. (1998). Attentional disruption is enhanced by the threat of pain. Behaviour Research and Therapy, 36, 195-204.

D'Esposito, M. (2007). From cognitive to neural models of working memory. Philosophical Transactions of the Royal Society B, 362, 761-772. doi:10.1098/rstb.2007.2086

Deco, G., \& Rolls, E. T. (2005). Neurodynamics of biased competition and cooperation for attention: A model with spiking neurons. Journal of Neurophysiology, 94, 295-313.

Dowman, R. (1994). SEP topographies elicited by innocuous and noxious sural nerve stimulation: II. Effects of stimulus intensity on topographic pattern and amplitude. Electroencephalography and Clinical Neurophysiology, 92, 303-315.

Dowman, R. (2001). Attentional set effects on human innocuous somatosensory and pain pathways. Psychophysiology, 38, 451-464.

Dowman, R. (2004). Electrophysiological indices of orienting attention towards pain. Psychophysiology, 41, 749-761.

Dowman, R. (2007a). Neural mechanisms of detecting and orienting attention towards unattended threatening somatosensory targets: I. Modality effects. Psychophysiology, 44, 407-419.
Dowman, R. (2007b). Neural mechanisms of detecting and orienting attention towards unattended threatening somatosensory targets: II. Intensity effects. Psychophysiology, 44, 420-430.

Dowman, R. (2014). Neural mechanisms underlying pain's ability to reorient attention: Evidence for sensitization of somatic threat detectors. Cognitive, Affective, \& Behavioral Neuroscience, 14, 805-817. doi:10.3758/s13415-013-0233-z

Dowman, R., \& ben-Avraham, D. (2008). An artificial neural network model of orienting attention towards threatening somatosensory stimuli. Psychophysiology, 45, 229-239.

Dowman, R., \& Darcey, T. M. (1994). SEP topographies elicited by innocuous and noxious sural nerve stimulation: III. Dipole source analysis. Electroencephalography and Clinical Neurophysiology, 92, 373-391

Dowman, R., Darcey, T. M., Barkan, H., Thadani, V., \& Roberts, D. (2007). Human intracranially-recorded cortical responses evoked by painful electrical stimulation of the sural nerve. NeuroImage, $34,743-763$.

Dowman, R., Glebus, G., \& Shinners, L. (2006). Effects of response conflict on pain-evoked medial prefrontal cortex activity. Psychophysiology, 42, 555-558.

Dowman, R., \& Schell, S. (1999). Innocuous-related sural nerve-evoked activity and finger-evoked potentials generated in the primary somatosensory and supplementary motor cortices. Clinical Neurophysiology, 110, 2104-2116.

Eccleston, C. (1994). Chronic pain and attention: A cognitive approach British Journal of Clinical Psychology, 33, 535-547.

Eccleston, C., \& Crombez, G. (1999). Pain demands attention: A cognitive-affective model of the interruptive function of pain. Psychological Bulletin, 125, 356-366. doi:10.1037/0033-2909. 125.3.356

Egner, T., Monti, J. M., \& Summerfield, C. (2010). Expectation and surprise determine neural population responses in the ventral visual stream. Journal of Neuroscience, 30, 16601-16608.

Escera, C., \& Corral, M. J. (2007). Role of mismatch negativity and novelty-P3 in involuntary auditory attention. Journal of Psychophysiology, 21, 251-264.

Friedman, D., Cycowicz, Y. M., \& Gaeta, H. (2001). The novelty P3: An event-related brain potential (ERP) sign of the brain's evaluation of novelty. Neuroscience \& Biobehavioral Reviews, 25, 355-373. doi: 10.1016/S0149-7634(01)00019-7

Friston, K. (2005). A theory of cortical responses. Philosophical Transactions of the Royal Society B, 360, 815-836.

Gilzenrat, M. S., Nieuwenhuis, S., Jepma, M., \& Cohen, J. D. (2010). Pupil diameter tracks changes in control state predicted by the adaptive gain theory of locus coeruleus function. Cognitive, Affective, \& Behavioral Neuroscience, 10, 252-269. doi:10.3758/CABN.10.2.252

Goldberg, Y. P., Pimstone, S. N., Namdari, R., Price, N., Cohen, C., Sherrington, R. P., \& Hayden, M. R. (2012). Human Mendelian pain disorders: A key to discovery and validation of novel analgesics. Clinical Genetics, 82, 367-373.

Halgren, E., Marinkovic, K., \& Chauvel, P. (1998). Generators of the late cognitive potentials in auditory and visual oddball tasks. Electroencephalography and Clinical Neurophysiology, 106, 156164.

Hsiao, S. S., O'Shaughnessy, D. M., \& Johnson, K. O. (1993). Effects of selective attention on spatial form processing in monkey primary and somatosensory cortex. Journal of Neurophysiology, 70, 444- 447.

International Association for the Study of Pain Task Force on Taxonomy. (1994). Classification of chronic pain: Descriptions of chronic pain syndromes and definitions of pain terms (2nd ed.). Seattle, WA: IASP Press.

Kastner, S., \& Ungerleider, L. G. (2000). Mechanisms of visual attention in the human cortex. Annual Reviews of Neuroscience, 23, 315-341. doi:10.1146/annurev.neuro.23.1.315 
Knight, R. T. (1996). Contribution of human hippocampal region to novelty detection. Nature, 383, 256-259.

Lavie, N. (2005). Distracted and confused? Selective attention under load. Trends in Cognitive Sciences, 9, 75-82. doi:10.1016/j.tics. 2004.12.004

Legrain, V., Iannetti, G. D., Plaghki, L., \& Mouraux, A. (2011). The pain matrix reloaded: A salience detection system for the body. Progress in Neurobiology, 93, 111-124. doi:10.1016/j.pneurobio.2010.10.005

Makeig, S., Debener, S., Onton, J., \& Delorme, A. (2004). Mining eventrelated brain dynamics. Trends in Cognitive Sciences, 8, 204-210.

Miller, E. K., \& Cohen, J. D. (2001). An integrative theory of prefrontal cortex function. Annual Review of Neuroscience, 24, 167-202. doi: 10.1146/annurev.neuro.24.1.167

Miron, D., Duncan, G. H., \& Bushnell, M. C. (1989). Effects of attention and unpleasantness of thermal pain. Pain, 39, 345-352.

Müller, H. J., \& Rabbit, P. M. A. (1989). Reflexive and voluntary orienting of visual attention: Time course of activation and resistance to interruption. Journal of Experimental Psychology: Human Perception and Performance, 15, 315-330. doi:10.1037/00961523.15.2.315

Nelder, J. A., \& Mead, R. (1965). A simple method for function minimization. Computer Journal, 7, 308-313. doi:10.1093/comjnl/7.4.308

Nieuwenhuis, S., Aston-Jones, G., \& Cohen, J. D. (2005). Decision making, the P3 and the locus-coeruleus-norepinephrine system. Psychological Bulletin, 131, 510-532. doi:10.1037/0033-2909. 131.4.510

Nieuwenhuis, S., De Geus, E. J., \& Aston-Jones, G. (2011). The anatomical and functional relationship between the $\mathrm{P} 3$ and autonomic components of the orienting response. Psychophysiology, 48, 162-175.

Norman, D. A., \& Shallice, T. (1986). Attention to action: Willed and automatic control of behavior. In R. J. Davison, G. E. Schwartz, \& D. Shapiro (Eds.), Consciousness and self-regulation: Advances in research (Vol. 4, pp. 1-18). New York, NY: Plenum Press.

Nunez, P. L. (1981). Electric fields of the brain. New York, NY: Oxford University Press.

Perez, M., \& Cohen, L. G. (2009). Interhemispheric inhibition between primary motor cortices: What have we learned? Journal of Physiology, 587, 725-726.

Polich, J. (2003). Theoretical overview of P3a and P3b. In J. Polich (Ed.), Detection of change: Event-related potential and fMRI findings (pp. 83-98). Boston, MA: Kluwer Academic Press.

Polich, J. (2007). Updating P300: An integrative theory of P3a and P3b. Clinical Neurophysiology, 118, 2128-2148.

Poranen, A., \& Hyvarinen, J. (1982). Effects of attention on multiunit responses to vibration in the somatosensory regions of the monkey's brain. Electroencephalography and Clinical Neurophysiology, 53, $525-537$.

Prinzmetal, W., McCool, C., \& Park, S. (2005). Attention: Reaction time and accuracy reveal different mechanisms. Journal of Experimental Psychology: General, 134, 73-92. doi:10.1037/0096-3445.134.1.73
Sara, S. J. (2009). The locus coeruleus and noradrenergic modulation of cognition. Nature Reviews Neuroscience, 10, 211-223.

Sara, S. J., \& Bouret, S. (2012). Orienting and reorienting: The locus coeruleus mediates cognition through arousal. Neuron, 76, 130-141.

Scherg, M. (1990). Fundamentals of dipole source potential analysis. In G. Grandori, M. Hoke, \& G. L. Romani (Eds.), Auditory evoked magnetic and electric potentials (pp. 40-69). Basel, The Netherlands: Karger.

Seidl, K. N., Peelen, M. V., \& Kastner, S. (2012). Neural evidence for distracter suppression during visual search in real-world scenes. Journal of Neuroscience, 32, 11812-11819. doi:10.1523/ JNEUROSCI.1693-12.2012

Shimamura, A. P. (2000). The role of the prefrontal cortex in dynamic filtering. Psychobiology, 28, 207-218.

Soltani, M., \& Knight, R. T. (2000). Neural origins of the P300. Critical Reviews in Neurobiology, 14, 199-224.

Summerfield, C., \& Egner, T. (2009). Expectation (and attention) in visual cognition. Trends in Cognitive Sciences, 13, 403-409.

Sylvester, C. M., Shulman, G. L., Jack, A. I., \& Corbetta, M. (2009). Anticipatory and stimulus-evoked blood oxygenation leveldependent modulations related to spatial attention reflect common additive signal. Journal of Neuroscience, 29, 10671-10682.

Van Damme, S., Crombez, G., Van Nieuwenborgh-De Wever, K., \& Goubert, L. (2008). Is distraction less effective when pain is threatening? An experimental investigation with the cold pressor task. European Journal of Pain, 12, 60-67.

Van Damme, S., Legrain, V., Vogt, J., \& Crombez, G. (2010). Keeping pain in mind: A motivational account of attention to pain. Neuroscience \& Biobehavioral Reviews, 34, 204-213. doi:10. 1016/j.neubiorev.2009.01.005

Vancleef, L. M. G., \& Peters, M. L. (2006). The interruptive effect of pain on attention. The Journal of Pain, 7, 21-22.

Wacongne, C., Changeux, J.-P., \& Dehaene, S. (2012). A neuronal model of predictive coding accounting for the mismatch negativity. Journal of Neuroscience, 32, 3665-3678.

Walsh, B. J., Buonocore, M. H., Cameron, C. S., \& Mangun, G. R. (2011). Integrating conflict detection and attentional control mechanisms. Journal of Cognitive Neuroscience, 23, 2211-2221.

Wessel, J. R., \& Aron, A. R. (2015). It's not too late: The onset of the frontocentral P3 indexes successful response inhibition in the stopsignal paradigm. Psychophysiology, 52, 472-480.

Yeung, N., Botvinick, M. W., \& Cohen, J. D. (2004). The neural basis of error detection: Conflict monitoring and the error-related negativity. Psychological Review, 111, 931-959. doi:10.1037/0033-295X.111. 4.931

Zhang, Z., \& Jewett, D. L. (1993). Insidious errors in dipole source localization parameters at a single time point due to model misspecification of number of shells. Electroencephalography and Clinical Neurophysiology, 88, 1-11. 